\title{
$K^{* 0}$ and $\phi$ Meson Production in Proton-Nucleus Interactions at $\sqrt{s}=41.6 \mathrm{GeV}$
}

I. $\mathrm{Abt}^{23}$, M. Adams ${ }^{10}$, M. Agari ${ }^{13}$, H. Albrecht ${ }^{12}$, A. Aleksandrov ${ }^{29}$, V. Amaral ${ }^{8}$, A. Amorim $^{8}$, S. J. Aplin ${ }^{12}$, V. Aushev ${ }^{16}$, Y. Bagaturia ${ }^{12,36}$, V. Balagura ${ }^{22}$, M. Bargiotti ${ }^{6}$, O. Barsukova ${ }^{11}$, J. Bastos ${ }^{8}$, J. Batista ${ }^{8}$, C. Bauer ${ }^{13}$, Th. S. Bauer ${ }^{1}$, A. Belkov ${ }^{11, \dagger}$, Ar. Belkov ${ }^{11}$, I. Belotelov ${ }^{11}$, A. Bertin ${ }^{6}$, B. Bobchenko ${ }^{22}$, M. Böcker ${ }^{26}$, A. Bogatyrev ${ }^{22}$, G. Bohm ${ }^{29}$, M. Bräuer ${ }^{13}$, M. Bruinsma ${ }^{28,1}$, M. Bruschi ${ }^{6}$, P. Buchholz ${ }^{26}$, T. Buran ${ }^{24}$, J. Carvalho ${ }^{8}$, P. Conde ${ }^{2,12}$, C. Cruse ${ }^{10}$, M. Dam ${ }^{9}$, K. M. Danielsen ${ }^{24}$, M. Danilov ${ }^{22}$, S. De Castro ${ }^{6}$, H. Deppe ${ }^{14}$, X. Dong ${ }^{3}$, H. B. Dreis ${ }^{14}$, V. Egorytchev ${ }^{12}$, K. Ehret $^{10}$, F. Eisele ${ }^{14}$, D. Emeliyanov ${ }^{12}$, S. Essenov ${ }^{22}$, L. Fabbri ${ }^{6}$, P. Faccioli ${ }^{6}$, M. Feuerstack-Raible ${ }^{14}$, J. Flammer ${ }^{12}$, B. Fominykh ${ }^{22}$, M. Funcke ${ }^{10}$, Ll. Garrido ${ }^{2}$, A. Gellrich ${ }^{29}$, B. Giacobbe ${ }^{6}$, J. Gläß $\AA^{20}$, D. Goloubkov ${ }^{12,33}$, Y. Golubkov ${ }^{12,34}$, A. Golutvin ${ }^{22}$, I. Golutvin ${ }^{11}$, I. Gorbounov ${ }^{12,26}$, A. Gorišek ${ }^{17}$, O. Gouchtchine ${ }^{22}$, D. C. Goulart ${ }^{7}$, S. Gradl ${ }^{14}$, W. Gradl ${ }^{14}$, F. Grimaldi ${ }^{6}$, J. Groth-Jensen ${ }^{9}$, Yu. Guilitsky ${ }^{22,35}$, J. D. Hansen ${ }^{9}$, J. M. Hernández ${ }^{29}$, W. Hofmann ${ }^{13}$, M. Hohlmann ${ }^{12}$, T. Hott ${ }^{14}$, W. Hulsbergen ${ }^{1}$, U. Husemann ${ }^{26}$, O. Igonkina ${ }^{22}$, M. Ispiryan ${ }^{15}$, T. Jagla ${ }^{13}$, C. Jiang ${ }^{3}$, H. Kapitza ${ }^{12}$, S. Karabekyan ${ }^{25}$, N. Karpenko ${ }^{11}$, S. Keller ${ }^{26}$, J. Kessler ${ }^{14}$, F. Khasanov ${ }^{22}$, Yu. Kiryushin ${ }^{11}$, I. Kisel ${ }^{23}$, E. Klinkby ${ }^{9}$, K. T. Knöpfle ${ }^{13}$, H. Kolanoski ${ }^{5}$, S. Korpar ${ }^{21,17}$, C. Krauss ${ }^{14}$, P. Kreuzer ${ }^{12,19}$, P. Križan ${ }^{18,17}$, D. Krücker ${ }^{5}$, S. Kupper ${ }^{17}$, T. Kvaratskheliia ${ }^{22}$, A. Lanyov ${ }^{11}$, K. Lau ${ }^{15}$, B. Lewendel ${ }^{12}$, T. Lohse ${ }^{5}$, B. Lomonosov ${ }^{12,32}$,

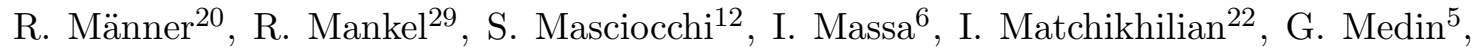
M. Medinnis ${ }^{12}$, M. Mevius ${ }^{12}$, A. Michetti ${ }^{12}$, Yu. Mikhailov ${ }^{22,35}$, R. Mizuk ${ }^{22}$, R. Muresan ${ }^{9}$, M. zur Nedden ${ }^{5}$, M. Negodaev ${ }^{12,32}$, M. Nörenberg ${ }^{12}$, S. Nowak ${ }^{29}$, M. T. Núñez Pardo de Vera ${ }^{12}$, M. Ouchrif ${ }^{28,1}$, F. Ould-Saada ${ }^{24}$, C. Padilla ${ }^{12}$, D. Peralta ${ }^{2}$, R. Pernack ${ }^{25}$, R. Pestotnik ${ }^{17}$, B. AA. Petersen ${ }^{9}$, M. Piccinini ${ }^{6}$, M. A. Pleier ${ }^{13}$, M. Poli ${ }^{6,31}$, V. Popov ${ }^{22}$, D. Pose ${ }^{11,14}$, S. Prystupa ${ }^{16}$, V. Pugatch ${ }^{16}$, Y. Pylypchenko ${ }^{24}$, J. Pyrlik ${ }^{15}$, K. Reeves ${ }^{13}$, D. Reßing ${ }^{12}$, H. Rick ${ }^{14}$, I. Riu ${ }^{12}$, P. Robmann ${ }^{30}$, I. Rostovtseva ${ }^{22}$, V. Rybnikov ${ }^{12}$, F. Sánchez ${ }^{13}$, A. Sbrizzi ${ }^{1}$, M. Schmelling ${ }^{13}$, B. Schmidt ${ }^{12}$, A. Schreiner ${ }^{29}$, H. Schröder ${ }^{25}$, U. Schwanke ${ }^{29}$, A. J. Schwartz ${ }^{7}$, A. S. Schwarz ${ }^{12}$, B. Schwenninger ${ }^{10}$, B. Schwingenheuer ${ }^{13}$, F. Sciacca ${ }^{13}$, N. SempriniCesari $^{6}$, S. Shuvalov ${ }^{22,5}$, L. Silva ${ }^{8}$, L. Sözüer ${ }^{12}$, S. Solunin ${ }^{11}$, A. Somov ${ }^{12}$, S. Somov ${ }^{12,33}$, J. Spengler ${ }^{12}$, R. Spighi ${ }^{6}$, A. Spiridonov ${ }^{29,22}$, A. Stanovnik ${ }^{18,17}$, M. Starič ${ }^{17}$, C. Stegmann ${ }^{5}$, H. S. Subramania ${ }^{15}$, M. Symalla ${ }^{12,10}$, I. Tikhomirov ${ }^{22}$, M. Titov ${ }^{22}$, I. Tsakov ${ }^{27}$, U. Uwer ${ }^{14}$, C. van Eldik ${ }^{12,10}$, Yu. Vassiliev ${ }^{16}$, M. Villa ${ }^{6}$, A. Vitale ${ }^{6}$, I. Vukotic ${ }^{5,29}$, H. Wahlberg ${ }^{28}$, A. H. Walenta ${ }^{26}$, M. Walter ${ }^{29}$, J. J. Wang ${ }^{4}$, D. Wegener ${ }^{10}$, U. Werthenbach ${ }^{26}$, H. Wolters ${ }^{8}$, R. Wurth ${ }^{12}$, A. Wurz ${ }^{20}$, Yu. Zaitsev ${ }^{22}$, M. Zavertyaev ${ }^{12,13,32}$, T. Zeuner ${ }^{12,26}$, A. Zhelezov ${ }^{22}$, Z. Zheng ${ }^{3}$, R. Zimmermann ${ }^{25}$, T. Živko ${ }^{17}$, and A. Zoccoli ${ }^{6}$

${ }^{1}$ NIKHEF, 1009 DB Amsterdam, The Netherlands ${ }^{a}$

${ }^{2}$ Department ECM, Faculty of Physics, University of Barcelona, E-08028 Barcelona, Spain ${ }^{b}$

${ }^{3}$ Institute for High Energy Physics, Beijing 100039, P.R. China

${ }^{4}$ Institute of Engineering Physics, Tsinghua University, Beijing 100084, P.R. China

${ }^{5}$ Institut für Physik, Humboldt-Universität zu Berlin, D-12489 Berlin, Germany ${ }^{c, d}$

${ }^{6}$ Dipartimento di Fisica dell' Università di Bologna and INFN Sezione di Bologna, I-40126 Bologna, Italy

${ }^{7}$ Department of Physics, University of Cincinnati, Cincinnati, Ohio 45221, USA ${ }^{e}$

${ }^{8}$ LIP Coimbra, P-3004-516 Coimbra, Portugal ${ }^{f}$

${ }^{9}$ Niels Bohr Institutet, DK 2100 Copenhagen, Denmark ${ }^{g}$ 
${ }^{10}$ Institut für Physik, Universität Dortmund, D-44221 Dortmund, Germany ${ }^{d}$

${ }^{11}$ Joint Institute for Nuclear Research Dubna, 141980 Dubna, Moscow region, Russia

${ }^{12}$ DESY, D-22603 Hamburg, Germany

${ }^{13}$ Max-Planck-Institut für Kernphysik, D-69117 Heidelberg, Germany ${ }^{d}$

${ }^{14}$ Physikalisches Institut, Universität Heidelberg, D-69120 Heidelberg, Germany ${ }^{d}$

${ }^{15}$ Department of Physics, University of Houston, Houston, TX 77204, USA ${ }^{e}$

${ }^{16}$ Institute for Nuclear Research, Ukrainian Academy of Science, 03680 Kiev, Ukraine ${ }^{h}$

${ }^{17}$ J. Stefan Institute, 1001 Ljubljana, Slovenia ${ }^{i}$

${ }^{18}$ University of Ljubljana, 1001 Ljubljana, Slovenia

${ }^{19}$ University of California, Los Angeles, CA 90024, USA ${ }^{j}$

${ }^{20}$ Lehrstuhl für Informatik V, Universität Mannheim, D-68131 Mannheim, Germany

${ }^{21}$ University of Maribor, 2000 Maribor, Slovenia

${ }^{22}$ Institute of Theoretical and Experimental Physics, 117259 Moscow, Russia ${ }^{k}$

${ }^{23}$ Max-Planck-Institut für Physik, Werner-Heisenberg-Institut, D-80805 München, Germany ${ }^{d}$

${ }^{24}$ Dept. of Physics, University of Oslo, N-0316 Oslo, Norway ${ }^{l}$

${ }^{25}$ Fachbereich Physik, Universität Rostock, D-18051 Rostock, Germany ${ }^{d}$

${ }^{26}$ Fachbereich Physik, Universität Siegen, D-57068 Siegen, Germany d

${ }^{27}$ Institute for Nuclear Research, INRNE-BAS, Sofia, Bulgaria

${ }^{28}$ Universiteit Utrecht/NIKHEF, 3584 CB Utrecht, The Netherlands ${ }^{a}$

${ }^{29}$ DESY, D-15738 Zeuthen, Germany

${ }^{30}$ Physik-Institut, Universität Zürich, CH-8057 Zürich, Switzerland ${ }^{m}$

${ }^{31}$ visitor from Dipartimento di Energetica dell' Università di Firenze and INFN Sezione di Bologna, Italy

${ }^{32}$ visitor from P.N. Lebedev Physical Institute, 117924 Moscow B-333, Russia

${ }^{33}$ visitor from Moscow Physical Engineering Institute, 115409 Moscow, Russia

${ }^{34}$ visitor from Moscow State University, 119899 Moscow, Russia

${ }^{35}$ visitor from Institute for High Energy Physics, Protvino, Russia

${ }^{36}$ visitor from High Energy Physics Institute, 380086 Tbilisi, Georgia

${ }^{\dagger}$ deceased

${ }^{a}$ supported by the Foundation for Fundamental Research on Matter (FOM), 3502 GA Utrecht, The Netherlands

${ }^{b}$ supported by the CICYT contract AEN99-0483

${ }^{c}$ supported by the German Research Foundation, Graduate College GRK 271/3

${ }^{d}$ supported by the Bundesministerium für Bildung und Forschung, FRG, under contract numbers 05-7BU35I, 05-7DO55P, 05-HB1HRA, 05-HB1KHA, 05-HB1PEA, 05-HB1PSA, 05-HB1VHA, 05-HB9HRA, 05-7HD15I, 057MP25I, 05-7SI75I

${ }^{e}$ supported by the U.S. Department of Energy (DOE)

${ }^{f}$ supported by the Portuguese Fundação para a Ciência e Tecnologia under the program POCTI

${ }^{g}$ supported by the Danish Natural Science Research Council

${ }^{h}$ supported by the National Academy of Science and the Ministry of Education and Science of Ukraine

${ }^{i}$ supported by the Ministry of Education, Science and Sport of the Republic of Slovenia under contracts number P1-135 and J1-6584-0106

${ }^{j}$ supported by the U.S. National Science Foundation Grant PHY-9986703

${ }^{k}$ supported by the Russian Ministry of Education and Science, grant SS-1722.2003.2, and the BMBF via the Max Planck Research Award

${ }^{l}$ supported by the Norwegian Research Council

$m$ supported by the Swiss National Science Foundation 


\begin{abstract}
The inclusive production cross sections of the strange vector mesons $K^{* 0}, \bar{K}^{* 0}$, and $\phi$ have been measured in interactions of $920 \mathrm{GeV}$ protons with $\mathrm{C}$, Ti, and $\mathrm{W}$ targets with the HERA-B detector at the HERA storage ring. Differential cross sections as a function of rapidity and transverse momentum have been measured in the central rapidity region and for transverse momenta up to $p_{T}=3.5 \mathrm{GeV} / \mathrm{c}$. The atomic number dependence is parametrised as $\sigma_{p A}=\sigma_{p N} * A^{\alpha}$, where $\sigma_{p N}$ is the proton-nucleon cross section. Within the phase space accessible, $\alpha\left(K^{* 0}\right)=0.86 \pm 0.03, \alpha\left(\bar{K}^{* 0}\right)=0.87 \pm 0.03$, and $\alpha(\phi)=0.96 \pm 0.02$. The total proton-nucleon cross sections, determined by extrapolating the differential measurements to full phase space, are $\sigma_{p N \rightarrow K^{* 0}}=(5.06 \pm 0.54) \mathrm{mb}, \sigma_{p N \rightarrow \bar{K}^{* 0}}=(4.02 \pm 0.45) \mathrm{mb}$, and $\sigma_{p N \rightarrow \phi}=(1.17 \pm 0.11) \mathrm{mb}$. For all resonances the Cronin effect is observed; compared to the measurements of Cronin et al. for $K^{ \pm}$mesons, the measured values of $\alpha$ for $\phi$ mesons coincide with those of $K^{+}$mesons for all transverse momenta, while the enhancement for $K^{* 0} / \bar{K}^{* 0}$ mesons is smaller.
\end{abstract}

Key words. Proton nucleus collision, hadronic interaction, vector meson production, Cronin effect, atomic number dependence

\title{
1 Introduction
}

The search for a deconfined state of quarks and gluons in relativistic heavy ion collisions is a subject which has received much attention in recent years $[1,2]$. It is a challenging task to find a signal that enables the discrimination of the short-lived locally deconfined state of quarks and gluons from a gas of confined hadrons. An enhancement of the strange quark fraction has been argued to appear in the quark gluon plasma [3]; hence one expects an increase of strange hadron production in these processes.

The rate of strange particle production depends on the temperature at which the hadronisation step takes place, and on the chemical potential. Hadronic interactions can influence the measurable strange particle rate in a two-fold way [4,5]: they can induce transitions to other states, and, in the case of hadrons with a short lifetime, rescattering of the final state particles can take place, affecting the reconstructed invariant mass signal $[6,7]$. Since the strength of these final state interactions depends strongly on the lifetime, $K^{* 0}$ and $\phi$ mesons - where the lifetimes differ by a factor of about 10 - have been proposed as a versatile tool to study these effects in relativistic heavy ion reactions.

Consequently, several new measurements of strange vector meson production have been published recently by RHIC experiments. Both for $K^{* 0}[8,9]$ and $\phi[10-16]$ mesons the influence of the very dense nuclear matter on the transverse momentum distributions and the line shape of the resonances have been studied in detail. In contrast to this, only limited information on $\phi$ production in proton-nucleus interactions exists up to now. The $A$-dependence of $\phi$ production in proton-nucleus interactions is studied in [17], while data in dAu collisions are available from the STAR and PHENIX collaborations $[14,18,19]$. No data are available on $K^{* 0}$ production.

Moreover, the study of the $p_{T}$ dependence of the inclusive production rate of $K^{* 0}$ and $\phi$ mesons in proton-nucleus (pA) interactions is of interest in its own right. Cronin et al. [20,21] observed a nuclear enhancement of high- $p_{T}$ particles in $\mathrm{pA}$ as compared to pp interactions for stable particles. In this paper we report the observation of the "Cronin effect" for the resonances $K^{* 0}, \bar{K}^{* 0}$, and $\phi$. The effect has been ascribed by many models to a broadening of the intrinsic transverse momentum of the primary partons in a hard scattering process [22]. The free parameters are fixed by fitting the predictions to hard pp interactions. The high $p_{T}$ meson spectra for proton-nucleus interactions are reasonably well described by this ansatz, which however fails to reproduce the measured high $p_{T}$ baryon spectra [23]. On the other hand, the differences between meson- and baryon-production in proton-nucleus reactions can be explained by a final-state recombination model [24]. Recently B.Z. Kopeliovich et al. [25] have formulated a parameter free 


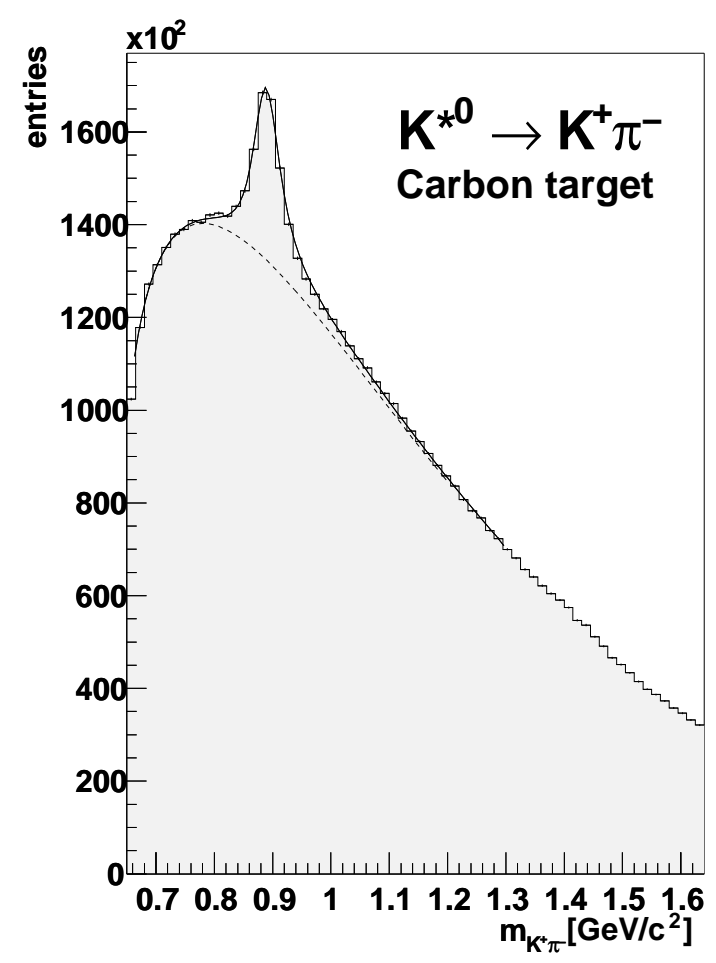

(a)

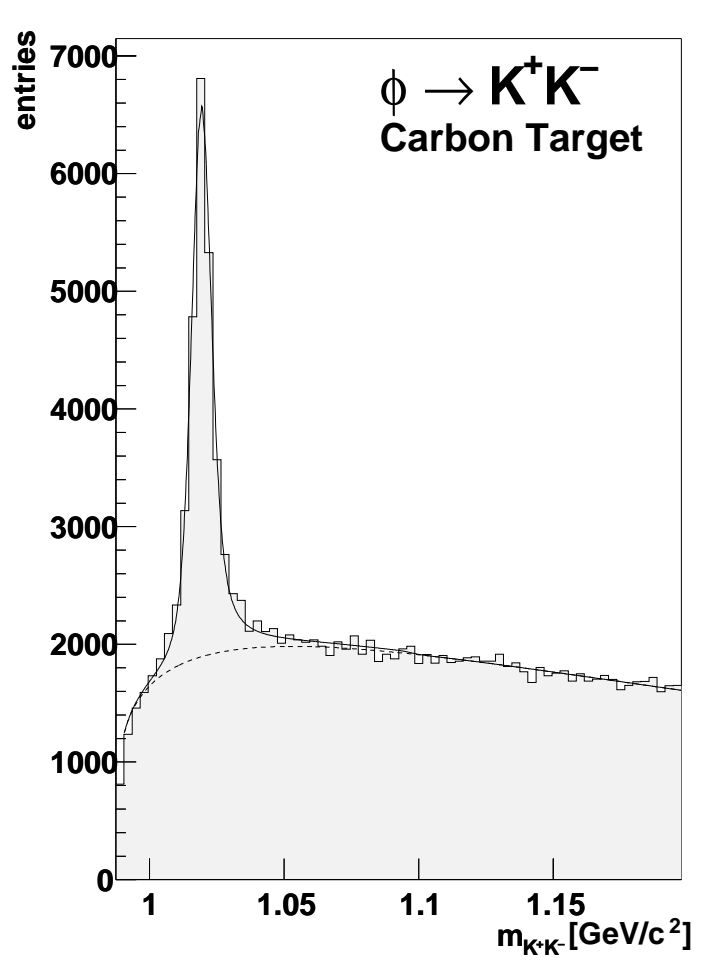

(b)

Figure 1. Invariant mass distributions for a) $K^{+} \pi^{-}$and b) $K^{+} K^{-}$mass combinations on the carbon target after application of the cuts described in the text. The distributions were fit by a Gaussian-smeared relativistic Breit-Wigner function and a background term (see text).

description of the process. It explains the Cronin effect as a result of a convolution of the initial parton distribution affected by initial state interactions, followed by the hard scattering process and the fragmentation of the scattered partons. From this ansatz, it follows that the steeper the $p_{T}$ dependence of the cross section, the larger the Cronin effect should be, i.e. its strength should depend on the flavor composition of the scattering partons via its structure- and fragmentation functions dependence. Moreover, a weak energy dependence of the Cronin effect is expected [25] in agreement with the observation of [21] for high- $p_{T} K^{ \pm}$mesons produced in proton-nucleus interactions in the energy range $200 \mathrm{GeV}$ to $400 \mathrm{GeV}$.

None of these models explicitly address the issue of the dependence of the Cronin effect on the flavor or mass of the produced hadron. The measurement presented here should motivate and aid further theoretical efforts to understand the Cronin effect and thereby lead to a deeper understanding of nuclear effects in pA collisions, particularly in the area of strangeness production.

\section{Data acquisition and event reconstruction}

The data were collected with the HERA-B detector operated at the proton ring of the HERA storage ring complex. The pN center-of-mass energy was $\sqrt{s}=41.6 \mathrm{GeV}$. The $K^{* 0} / \bar{K}^{* 0}$ data cover the rapidity interval $-0.8 \leq y \leq 0.3$ (in the centre-of-mass system) and a transverse momentum range of $0 \leq p_{T}^{2} \leq 12 \mathrm{GeV}^{2} / \mathrm{c}^{2}$. The $\phi$ results were obtained for $-0.7 \leq y \leq 0.25$ and $0.3 \leq p_{T}^{2} \leq 12 \mathrm{GeV}^{2} / \mathrm{c}^{2}$. Here the lack of data for $p_{T}^{2}<0.3 \mathrm{GeV}^{2} / \mathrm{c}^{2}$ is due to the small 
opening angle of the $\phi$ decay in the lab system that makes the decay products escape through the beam-pipe.

The HERA-B detector is a forward magnetic spectrometer with a large acceptance for particles produced in the central region. The most important components of the detector for this analysis are a wire target [26] inserted into the halo of the $920 \mathrm{GeV}$ proton beam, a vertex detector [27] followed by a magnetic dipole field of $2.13 \mathrm{Tm}$, a tracking system [28] using drift tube chambers, a Ring Imaging Cherenkov detector (RICH) [29] which enables the identification of $K^{ \pm}$mesons above a threshold of $9.6 \mathrm{GeV} / \mathrm{c}$, and an electromagnetic calorimeter [30]. A more detailed overview of the HERA-B detector can be found elsewhere [31].

The analysis is based on data recorded in the run period 2002/03. From a total of $200 \cdot 10^{6}$ events collected with three different target materials a sub-sample of $132 \cdot 10^{6}$ events taken in a 2 -weeks period in December 2002 was selected to minimise systematic uncertainties. About $58 \cdot 10^{6}$ events were recorded with a carbon $(\mathrm{C})$ wire target, $21 \cdot 10^{6}$ events with a titanium (Ti) target, and $53 \cdot 10^{6}$ events with a tungsten $(\mathrm{W})$ target. As the data were recorded at a moderate interaction rate of $1.5 \times 10^{6} \mathrm{~s}^{-1}$, compared to an average rate of filled proton bunches of $8.5 \times 10^{6} \mathrm{~s}^{-1}$ at the target, only about $18 \%$ of all bunch crossings with the target produce an inelastic interaction. To reject empty bunch crossings an interaction trigger accepted only those events, where either the number of hits in the RICH was $>20$ (for a $\beta=1$ particle 32 photons are detected on average), or a cluster with an energy $>1 \mathrm{GeV}$ was detected in the inner part of the electromagnetic calorimeter. The efficiency of the trigger was larger than $99 \%$ [32]. In parallel to the interaction trigger, events were selected in a random manner, regardless of whether or not they contained an inelastic interaction. These random events were used to study possible biases of the interaction trigger [32].

The events were reconstructed with the standard HERA-B analysis package [33], based on information from the vertex detector and the tracking system. Charged particles were identified by matching the tracks with rings reconstructed in the RICH. Using the track momentum information, the likelihood probability for charged kaons, protons, light particles $\left(e, \mu, \pi^{ \pm}\right)$, and a background hypothesis were calculated [29].

The integrated luminosity was determined from the interaction rate at the target monitored by various devices and from the total inelastic cross section [32].

\section{Data analysis}

\section{$3.1 K^{ \pm}$meson identification and $K^{* 0}(\phi)$ optimisation}

To identify $K^{* 0} / \bar{K}^{* 0}$ and $\phi$, the decay channels $K^{* 0} \rightarrow K^{+} \pi^{-}, \bar{K}^{* 0} \rightarrow K^{-} \pi^{+}$, and $\phi \rightarrow K^{+} K^{-}$ were investigated. For all events with at least two oppositely charged tracks the invariant mass $m_{i n v}$ of the pair was separately calculated for the $K^{+} \pi^{-}, K^{-} \pi^{+}$, and $K^{+} K^{-}$mass assumption. For the further analysis mass combinations were accepted if

$$
0.63 \mathrm{GeV} / \mathrm{c}^{2} \leq m_{\text {inv }} \leq 1.6 \mathrm{GeV} / \mathrm{c}^{2}
$$

for the $K^{ \pm} \pi^{\mp}$, and

$$
0.988 \mathrm{GeV} / \mathrm{c}^{2} \leq m_{\text {inv }} \leq 1.198 \mathrm{GeV} / \mathrm{c}^{2}
$$

for the $K^{+} K^{-}$combinations, respectively. In order to reduce the combinatoric background, $K^{ \pm}$mesons were identified by the RICH. For the $K^{* 0}\left(\bar{K}^{* 0}\right)$ candidates, the kaon identification was demanded for the positive (negative) track, while for the $\phi$ candidates kaon identification was applied for both tracks. Only $K^{ \pm}$candidates with a momentum $p_{K} \geq 9.7 \mathrm{GeV} / \mathrm{c}$ were considered, slightly above the kaon threshold of the RICH. While for the determination of the 
$\phi$ signal a soft likelihood probability cut of $\mathcal{L}_{K} \geq 0.3$ efficiently suppressed the background, a harder cut of $\mathcal{L}_{K} \geq 0.95$ was required for the $K^{* 0}$ to reduce the combinatoric background. The non-existence of mass reflections has been checked by experimental data and by the Monte-Carlo (MC) simulation described in chapter 3.3 .

For momenta $p_{K}<40 \mathrm{GeV} / \mathrm{c}$ the efficiency for identification of $K^{ \pm}$mesons is high, amounting to about $90 \%$ [29]. For higher momenta the discrimination power drops and the misidentification probability increases, since for such momenta the Cherenkov angles for $\pi$ and $K$ mesons become indistinguishable. The $K$ meson identification efficiency as a function of $K$ momentum has been determined by MC. It was checked with $\phi \rightarrow K^{+} K^{-}$data [34] by identifying either one or both kaons and reconstructing the $\phi$ resonance. The difference in efficiency between data and MC of $5 \%$ per identified kaon was corrected for, and a residual systematic error of $2 \%$ was estimated (see chapter 3.4). A large fraction of the produced $K^{* 0}$ and $\phi$ candidates were rejected by the momentum cut $p_{K} \geq 9.7 \mathrm{GeV} / \mathrm{c}$. The total efficiency for $K$ mesons from $K^{* 0}$ and $\phi$ resonance decays to survive the RICH identification and the momentum cut was about $15 \%$.

The significance of the signal was improved further by applying soft track quality cuts. In case of the vertex detector at least 6 hits per track were demanded. In the tracking system a cut of at least 15 hits per track was used to enhance the significance of the $K^{* 0}(\phi)$ signal without reducing the efficiency. On average, a track comprised 12 hits in the vertex detector and 40 hits in the tracking system, respectively. The $K^{* 0}$ signal significance was improved further by applying a cut of $p_{\pi} \geq 2 \mathrm{GeV} / \mathrm{c}$ to pion track candidates [35]. The cuts were optimised for significance using signal $\mathrm{MC}$ and real data background events.

\subsection{Signal determination}

Typical invariant mass distributions for the $K^{+} \pi^{-}$and $K^{+} K^{-}$combinations are shown in Fig. 1. after application of the cuts mentioned above. To facilitate the extraction of the signal, the resonance was described by a relativistic $P$-wave Breit-Wigner [36]:

$$
\begin{aligned}
B W(m) & =\frac{m \cdot m_{0} \cdot \Gamma(m)}{\left(m_{0}^{2}-m^{2}\right)^{2}+\left(m_{0} \cdot \Gamma(m)\right)^{2}} \\
\Gamma(m) & =\Gamma_{0} \cdot\left(\frac{q}{q_{0}}\right)^{2 l+1} \cdot \frac{m_{0}}{m},
\end{aligned}
$$

with

$$
\begin{aligned}
\Gamma_{0} & =\Gamma\left(m_{0}\right) \text { the natural width } \\
q(m)_{\mid K^{* 0}} & =\frac{\sqrt{\left(m^{2}-\left(m_{K}+m_{\pi}\right)^{2}\right)\left(m^{2}-\left(m_{K}-m_{\pi}\right)^{2}\right)}}{2 m} \\
q(m)_{\mid \phi} & =\frac{\sqrt{m^{2}-4 m_{K}^{2}}}{2} \\
q_{0} & =q\left(m_{0}\right) \\
l & =1 .
\end{aligned}
$$

$q(m)$ is the momentum of the decay products in the rest frame of the mother particle, and $l$ is the angular momentum transferred. To take into account effects of the detector resolution the Breit-Wigner distribution was numerically folded with a Gaussian of width $\sigma_{m}$ in the fits of the measured mass spectra. It has been checked that the mass resolutions in experimental data and $\mathrm{MC}$ are consistent within errors. The mass $m_{0}$ of the resonance and the width $\sigma_{m}$ were free parameters of the fit, while the natural width $\Gamma_{0}$ of the resonance was fixed to its PDG 
Table 1. Number of inelastic events $\left(N_{i a}\right)$, number of $K^{* 0}, \bar{K}^{* 0}$, and $\phi$ candidates $\left(N_{V M}\right)$ after analysis cuts, reconstructed mass $\left(m_{r e c}\right)$, and experimental mass resolution $\left(\sigma_{m}\right)$ for each target material. Errors are statistical only.

\begin{tabular}{|c|c|c|c|c|}
\hline & $N_{i a}$ & $N_{V M}$ & $m_{\text {rec }}\left[\mathrm{MeV} / c^{2}\right]$ & $\sigma_{m}\left[\mathrm{MeV} / c^{2}\right]$ \\
\hline \multicolumn{5}{|c|}{$p A \rightarrow K^{* 0} X$} \\
\hline $\mathrm{C}$ & $58 \cdot 10^{6}$ & $187574 \pm 1726$ & $890.5 \pm 0.2$ & $5.1 \pm 0.2$ \\
\hline $\mathrm{Ti}$ & $21 \cdot 10^{6}$ & $83231 \pm 1237$ & $890.7 \pm 0.4$ & $5.0 \pm 0.3$ \\
\hline W & $53 \cdot 10^{6}$ & $262356 \pm 2420$ & $890.8 \pm 0.2$ & $5.3 \pm 0.3$ \\
\hline \multicolumn{5}{|c|}{$p A \rightarrow \bar{K}^{* 0} X$} \\
\hline $\mathrm{C}$ & $58 \cdot 10^{6}$ & $149721 \pm 1622$ & $890.8 \pm 0.2$ & $4.6 \pm 0.1$ \\
\hline $\mathrm{Ti}$ & $21 \cdot 10^{6}$ & $65823 \pm 1147$ & $890.7 \pm 0.4$ & $5.1 \pm 0.4$ \\
\hline $\mathrm{W}$ & $53 \cdot 10^{6}$ & $195373 \pm 2225$ & $891.5 \pm 0.3$ & $4.8 \pm 0.2$ \\
\hline \multicolumn{5}{|c|}{$p A \rightarrow \phi X$} \\
\hline $\mathrm{C}$ & $58 \cdot 10^{6}$ & $15379 \pm 204$ & $1019.2 \pm 0.1$ & $2.6 \pm 0.1$ \\
\hline $\mathrm{Ti}$ & $21 \cdot 10^{6}$ & $7580 \pm 142$ & $1019.2 \pm 0.1$ & $2.7 \pm 0.1$ \\
\hline $\mathrm{W}$ & $53 \cdot 10^{6}$ & $26971 \pm 295$ & $1019.3 \pm 0.1$ & $2.6 \pm 0.1$ \\
\hline
\end{tabular}

Table 2. Summary of systematic errors for the integrated and total production cross section measurements. See text for a more detailed explanation. Numbers are grouped target material wise. The last two errors are due to the phase space extrapolation and have been applied only to the total cross sections. Numbers are given in $\%$.

\begin{tabular}{lccc|ccc|ccc}
\hline & \multicolumn{3}{c}{$\mathrm{C}$} & \multicolumn{3}{c}{$\mathrm{Ti}$} & \multicolumn{3}{c}{$\mathrm{W}$} \\
\hline & $K^{* 0}$ & $\bar{K}^{* 0}$ & $\phi$ & $K^{* 0}$ & $\bar{K}^{* 0}$ & $\phi$ & $K^{* 0}$ & $\bar{K}^{* 0}$ & $\phi$ \\
\hline MC statistics & 1.0 & 1.3 & 1.6 & 1.0 & 1.3 & 1.1 & 1.0 & 1.3 & 0.7 \\
Bg. parametrisation & 2.2 & 1.9 & - & 1.7 & 2.3 & - & 2.5 & 2.3 & - \\
Cut variation & 2.8 & 2.8 & 6.2 & 2.8 & 2.8 & 7.0 & 2.8 & 2.8 & 7.1 \\
BR & 0.1 & 0.1 & 1.22 & 0.1 & 0.1 & 1.22 & 0.1 & 0.1 & 1.22 \\
Luminosity & & 4.8 & & & 5.0 & & & 3.8 & \\
Luminosity scaling & & 2.0 & & & 2.0 & & & 2.0 \\
Track efficiency & & 3.0 & & & 3.0 & & & 3.0 & \\
\hline$p_{T}^{2}$-extr. & - & - & 6.6 & - & - & 8.8 & - & - & 8.2 \\
$y$-extr. & 2.6 & 4.1 & 6.3 & 2.6 & 4.5 & 6.0 & 2.6 & 4.5 & 4.9 \\
\hline
\end{tabular}

value [37]. The number of resonance decays was estimated by integrating the fitted signal in the interval $\pm 3 \Gamma_{0}$ around the resonance mass $m_{0}$. A variation of the fit interval by $\Gamma_{0}$ changed the yield up to $5 \%$. The same was observed for the MC used for acceptance correction, thus compensating the effect seen in data.

The background was parametrised by expressions which have been applied successfully in other experiments. For the $K^{* 0} / \bar{K}^{* 0}$ the parametrisation of [38] was adopted:

$$
B G_{K * 0}(m)=p_{0} \cdot\left(\frac{q}{m}\right)^{p_{1}} \cdot e^{-p_{2} q-p_{3} q^{2}}
$$

where $p_{0} \ldots p_{3}$ are free parameters and $q$ is the momentum transfer introduced above. For the $\phi$ meson the background was described by a parametrisation proposed in [39]:

$$
B G_{\phi}(m)=p_{0} \cdot m_{r e l}^{p_{1}} \cdot e^{-p_{2} m-p_{3} m^{2}},
$$

where $m_{r e l}=m-2 m_{K}$ and $p_{0} \ldots p_{3}$ again are free parameters of the fit. As shown in Fig. 1, these combinations of signal and background functions describe the data properly.

It has been shown $[34,35]$ that both the fitted masses and the detector resolution $\sigma_{m}$ derived from the data were stable for the whole data taking period and independent of the target 
material. Also the resonance yields, normalized to the integrated luminosity, were stable during the whole data taking period. Table 1 summarises the $K^{* 0}, \bar{K}^{* 0}$ and $\phi$ statistics obtained after cuts, the reconstructed mass positions, and the experimental mass resolutions.

The bin size for the $p_{T^{-}}^{2}$ and rapidity $(y$-) distributions were chosen to be broader than the resolution determined from MC simulation. From MC we find that in each bin more than $80 \%$ of the reconstructed vector mesons were generated in the same bin.

\subsection{Acceptance correction}

Acceptance correction functions were determined from MC events using FRITIOF 7.02 [40]. The detector simulation was based on GEANT 3.21 [41] and includes the measured hit resolutions, mapping of inefficient channels, and electronic noise. The simulated events were processed by the same reconstruction chain as the data. Since the FRITIOF generator uses an approximation for the generation of hard parton-parton scattering, the MC $p_{T}^{2}$ distributions show a steeper slope than observed in the data. To make sure that the reconstruction efficiencies and the influence of the analysis cuts are correctly described by the simulation, the MC events were reweighted in $p_{T}^{2}$ according to the data for both the $K^{* 0} / \bar{K}^{* 0}$ and the $\phi$ meson. It was checked that after reweighting both the rapidity and azimuthal MC distributions match those of the data.

The total efficiency for each $p_{T^{-}}^{2}$ and $y$-bin was determined with the help of the reweighted MC events by comparing the number of reconstructed and produced $K^{* 0} / \phi$ mesons in each bin. The number of reconstructed mesons was obtained similar to data by a fit to the reconstructed invariant mass spectra. Typical efficiencies vary between $5 \%$ and $30 \%$ [34,35]. Studies of systematic uncertainties due to signal extraction and background estimation show that they are negligible compared to the statistical error in the corresponding interval. Note that for each rapidity bin the efficiencies are determined by integrating over the measured $p_{T}^{2}$-range and vice versa.

\subsection{Systematic errors}

Possible sources of systematic uncertainties have been studied in detail: Since especially the $K^{* 0} / \bar{K}^{* 0}$ invariant mass distributions are affected by a large combinatorial background peaking close to the resonance signal, an alternative background parametrisation to (3) was used, which was proven to be successful in studies of $K^{* 0}$ production in $e^{+} e^{-}$-collisions [42]:

$$
B G(m)=p_{0}\left[1-\left(\frac{1}{m+p_{1}}\right)^{p_{2}}\right] \cdot\left(1+p_{3} m\right),
$$

where $p_{0} \ldots p_{3}$ are free parameters. The signal yields obtained in most $p_{T^{-}}^{2}$ and $y$-intervals agree for the two analyses within their statistical uncertainty. The absolute difference of the yields, derived using the two background parametrisations, is considered as a systematic error. It amounts to about $2 \%$ for the integrated cross sections. For the $\phi$ meson a corresponding study [34] provided much smaller deviations, and their contribution to the systematic error can be safely neglected.

The variation of the analysis cuts, including the uncertainties in the kaon identification efficiency $(2.0 \%$ per identified particle), resulted in a systematic uncertainty of $2.8 \%$ for the $K^{* 0} / \bar{K}^{* 0}$ and $6.2-7.1 \%$ for the $\phi$ analysis (depending on the target material). The systematic error of the luminosity consists of an overall scaling error of $2 \%$ and a target material dependent part of 3.8-5.0\%, and the systematic error of the branching ratios [37] amounts to $0.1 \%$ and $1.22 \%$ for $K^{* 0} \rightarrow K^{+} \pi^{-} / \bar{K}^{* 0} \rightarrow K^{-} \pi^{+}$and $\phi \rightarrow K^{+} K^{-}$, respectively. A systematic error on 


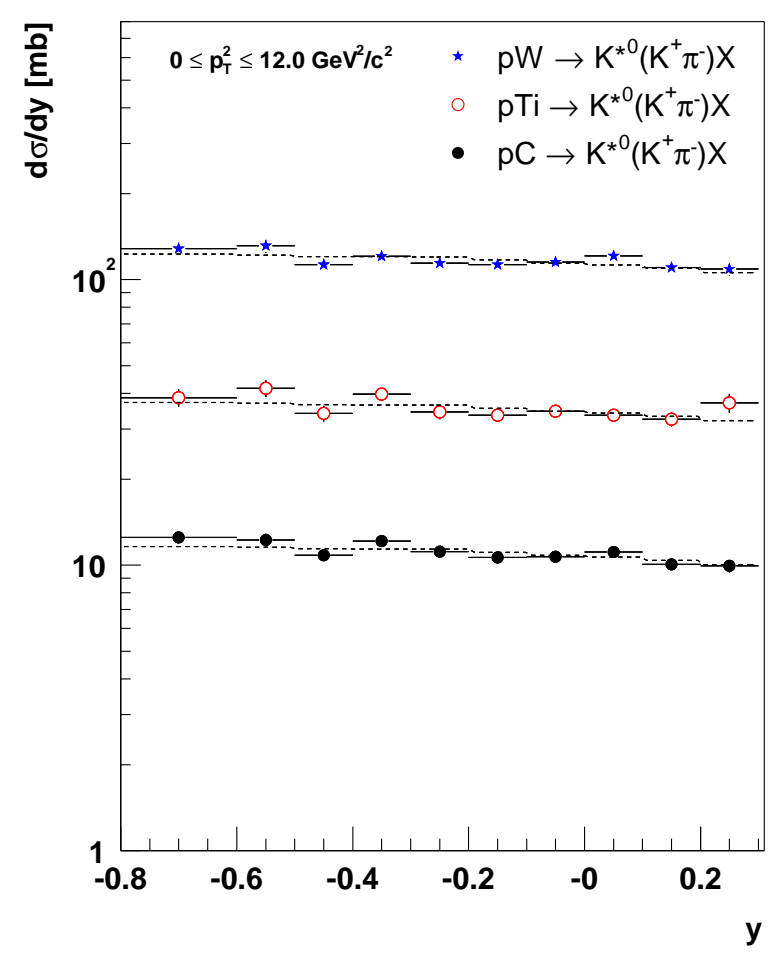

(a)

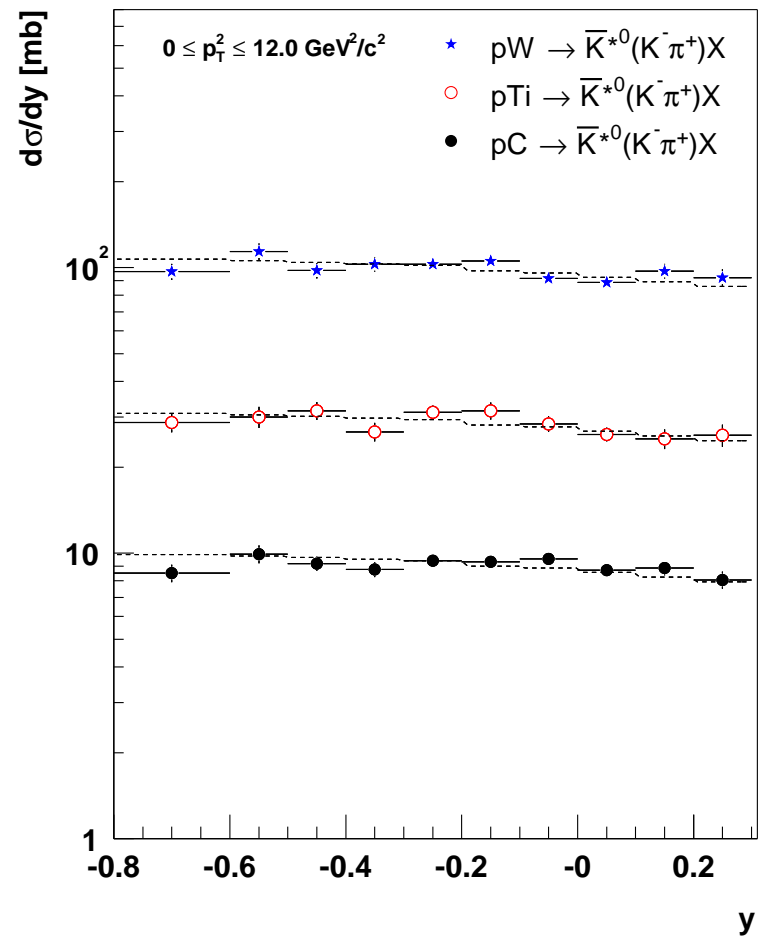

(b)

Figure 2. Measured inclusive differential cross sections $d \sigma / d y$ as a function of rapidity for a) $K^{* 0}$ and b) $\bar{K}^{* 0}$ meson production in the range $0 \leq p_{T}^{2} \leq 12 \mathrm{GeV}^{2} / \mathrm{c}^{2}$. Horizontal bars indicate the widths of the individual bins, while vertical errors bars represent the uncertainties due to statistics. Note that for most bins the error is smaller than the size of the symbols. The dashed lines show the (scaled) results of the FRITIOF MC.

the track efficiency of $1.5 \%$ per track was estimated using $K_{s}^{0} \rightarrow \pi^{+} \pi^{-}$decays [43]. A summary of all systematic errors is given in Table 2 .

\section{Experimental results}

\subsection{Differential cross sections}

Due to limited statistics, we did not calculate the double-differential cross section, but rather determined the one-dimensional rates $d N / d p_{T}^{2}$ and $d N / d y$, which were integrated over the measured $y$ and $p_{T}^{2}$ range, respectively. Given the corresponding one-dimensional efficiencies $\epsilon_{a c c}\left(p_{T}^{2}\right)$ and $\epsilon_{a c c}(y)$, the differential cross sections can be written as:

$$
\frac{d \sigma}{d x}=\frac{1}{B R \cdot \mathcal{L}} \frac{d N}{d x} \frac{1}{\epsilon_{a c c}(x)}
$$

where $x=\left\{y, p_{T}^{2}\right\} . B R$ and $\mathcal{L}$ denote the branching ratio of the decay and the integrated luminosity of the data set, respectively. The acceptance boundaries of the measurement in rapidity and transverse momentum squared are given in Table 4.

The measured cross sections for the $K^{* 0}$ and the $\bar{K}^{* 0}$ mesons as a function of rapidity are shown in Fig. 2, The cross sections are rather flat as expected in the central rapidity region [44]. The $\bar{K}^{* 0}$ cross sections are suppressed in comparison to the $K^{* 0}$ cross sections by about $20 \%$, in agreement with the results of the FRITIOF MC. The rapidity distributions for $\phi$ mesons 
produced on $\mathrm{C}, \mathrm{Ti}$, and $\mathrm{W}$ targets are shown in Fig. 4, Again the cross section in the central region is flat.

Transverse momentum spectra are shown in Fig. 3 for the $K^{* 0}$ and $\bar{K}^{* 0}$. The spectra deviate from a simple exponential shape at large transverse momenta, which can be traced back to hard parton-parton scattering [45]. Common exponential parametrisations like

$$
\frac{d \sigma}{d p_{T}^{2}}=C_{2} e^{-a p_{T}^{2}}
$$

or

$$
\frac{d \sigma}{d p_{T}^{2}}=C_{3} e^{-b m_{T}}, m_{T}=\sqrt{m_{0}^{2}+p_{T}^{2}}
$$

only fit the data for $p_{T}^{2} \lesssim 1 \mathrm{GeV}^{2} / \mathrm{c}^{2}$.

On the contrary, as is shown in Fig. 3, a power-law parametrisation,

$$
\frac{d \sigma}{d p_{T}^{2}}=C_{1}\left(1+\frac{p_{T}^{2}}{p_{0}^{2}}\right)^{-\beta}
$$

describes the spectra over the full $p_{T}^{2}$ range of the measurements. The $p_{T}^{2}$-spectrum of $\bar{K}^{* 0}$ mesons, which have no valence quarks in common with nucleons, is steeper than the $K^{* 0}$ meson spectrum. Similar observations have been made in hard pp collions for $K^{-}$mesons in comparison to $K^{+}$mesons $[45,46]$. In the latter case this observation has been traced back to the fact that $K^{-}$ mesons are, for the kinematic condition of the experiment, dominantly gluon fragments [45, 47]. QCD calculations [48] show that for a fixed value of $x_{T}=2 p_{T} / \sqrt{s}$ the relative contribution of gluon fragments to $K^{-}\left(\bar{K}^{* 0}\right)$ production decreases while the sea quark share is enhanced when the CMS energy is increased.

The same parametrisation (9) successfully fits the transverse momentum distributions for the $\phi$, shown in Fig. 5. The suppression of $\phi$ mesons as compared to $\bar{K}^{* 0}$ mesons by a factor of 3 to 4 is compatible with the known strangeness suppression in fragmentation processes [42]. No simple qualitative explanation exists for the observation that the $\bar{K}^{* 0} p_{T}$ spectrum is steeper than that of $\phi$ meson.

The fit parameters for all particles are collected in Table 3. Note that the $\chi^{2}$ of the tungsten fits is considerably worse than those of the other target materials. This is likely caused by an enhancement of background due to the large multiplicity of the pW interactions. The $\chi^{2}$ improves however considerably when leaving out the bin of largest $p_{T}^{2}$ in the fits. The differential cross sections $d \sigma / d y$ and $d \sigma / d p_{T}^{2}$ for $K^{* 0}, \bar{K}^{* 0}$, and $\phi$ production are summarised in Tables 6 and 7 ,

\subsection{Integrated inclusive and total cross sections}

The integrated cross sections in the acceptance region of the HERA-B detector are calculated by fitting the shape of the MC distributions to the differential cross sections $d \sigma / d y$. The results of the fits are shown as dashed lines in Figs. 2 and 4 . The integrated cross sections $\sigma_{v i s}$ in the phase space accessible to the HERA-B experiment are listed in Table 4. Note that integrating the $p_{T}^{2}$ differential distributions yields consistent cross sections.

The good agreement of the $K^{* 0}$ and $\bar{K}^{* 0}$ transverse momentum spectra with the power-law parametrisation (9) for small $p_{T}$ suggests that this is true also for the $\phi$ differential cross sections, where only data with $p_{T}^{2} \geq 0.3 \mathrm{GeV}^{2} / \mathrm{c}^{2}$ are available. Hence we have used this parametrisation to extrapolate the $\phi$ differential cross section to $0 \leq p_{T}^{2} \leq 0.3 \mathrm{GeV}^{2} / \mathrm{c}^{2}$ when calculating the total cross section. The extrapolation factors are summarised in Table 4 , The systematic uncertainty 


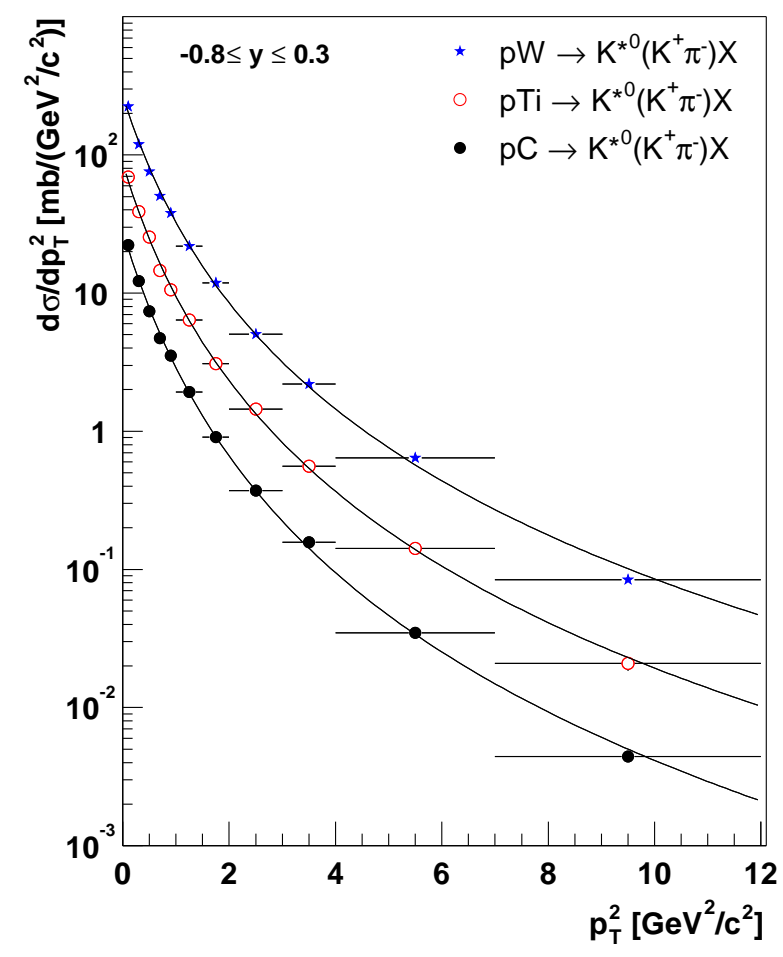

(a)

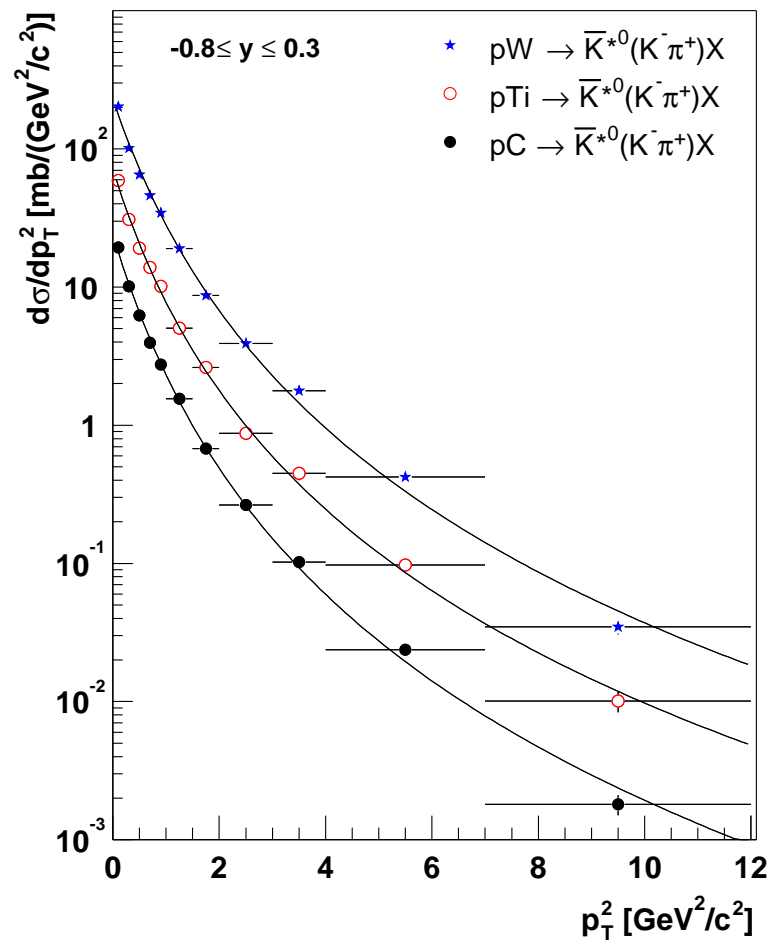

(b)

Figure 3. Measured inclusive differential cross sections $d \sigma / d p_{T}^{2}$ as a function of $p_{T}^{2}$ for a) $K^{* 0}$ and b) $\bar{K}^{* 0}$ production. The vertical error bars reflect statistical errors only. Fits to the parametrisation (9) are superimposed.

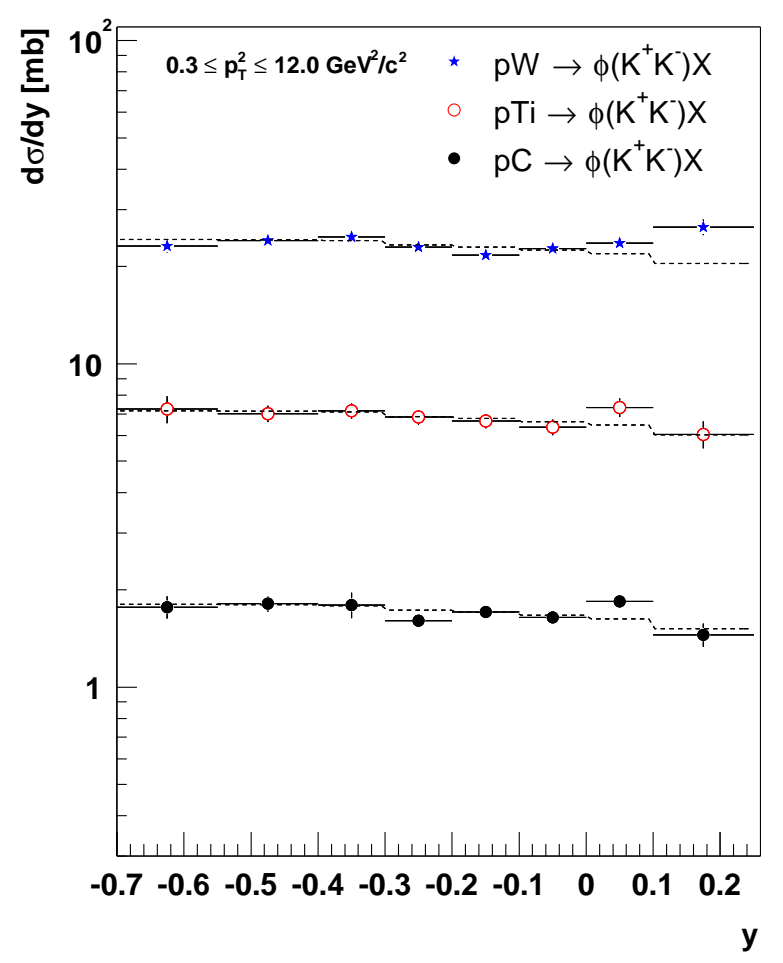

Figure 4. Measured inclusive differential cross section $d \sigma / d y$ as a function of rapidity for $\phi$ meson production in the range $0.3 \mathrm{GeV}^{2} / \mathrm{c}^{2} \leq p_{T}^{2} \leq 12 \mathrm{GeV}^{2} / \mathrm{c}^{2}$. The vertical error bars reflect the uncertainties due to statistics. The dashed lines show the (scaled) results of the FRITIOF MC. 


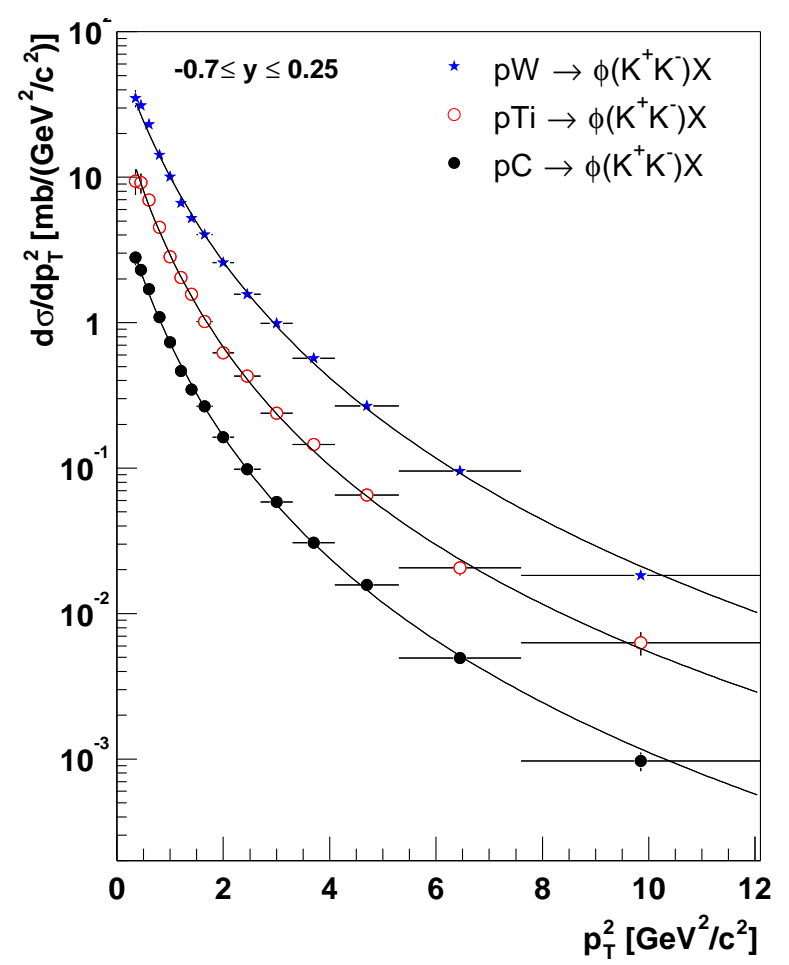

Figure 5. Measured inclusive differential cross sections $d \sigma / d p_{T}^{2}$ as a function of $p_{T}^{2}$ for $\phi$ meson production. The vertical error bars represent the uncertainties due to statistics. Fits to the parametrisation (9) are superimposed.

Table 3. Results of the power-law fits (9) for the differential cross sections $d \sigma / d p_{T}^{2}$. Fits were performed within the full $p_{T}^{2}$-range of the measurements and in the rapidity interval accessible by the experiment $(-0.8 \leq y \leq 0.3$ for $K^{* 0} / \bar{K}^{* 0},-0.7 \leq y \leq 0.25$ for $\left.\phi\right)$. The last column gives the mean $p_{T}$ of the cross sections, calculated from the fit parameters.

\begin{tabular}{cccccc}
\hline & $C_{1}\left[\mathrm{mb} / \mathrm{GeV}^{2}\right]$ & $\beta$ & $p_{0}^{2}\left[\mathrm{GeV}^{2} / \mathrm{c}^{2}\right]$ & $\chi^{2} /$ n.d.f. & $\left\langle p_{T}\right\rangle[\mathrm{GeV} / \mathrm{c}]$ \\
\hline \multicolumn{5}{c}{$p A \rightarrow K^{* 0} X$} \\
\hline $\mathrm{C}$ & $28.5 \pm 1.1$ & $4.17 \pm 0.12$ & $1.36 \pm 0.08$ & $9.1 / 8$ & $0.66 \pm 0.07$ \\
$\mathrm{Ti}$ & $87.1 \pm 5.2$ & $3.89 \pm 0.14$ & $1.30 \pm 0.11$ & $8.3 / 8$ & $0.69 \pm 0.09$ \\
$\mathrm{~W}$ & $268.5 \pm 10.9$ & $3.75 \pm 0.10$ & $1.32 \pm 0.08$ & $24.5 / 8$ & $0.72 \pm 0.07$ \\
\hline \multicolumn{5}{c}{$p A \rightarrow \bar{K}^{* 0} X$} \\
\hline $\mathrm{C}$ & $24.4 \pm 1.0$ & $4.67 \pm 0.16$ & $1.52 \pm 0.11$ & $14.2 / 8$ & $0.64 \pm 0.07$ \\
$\mathrm{Ti}$ & $71.9 \pm 4.4$ & $4.39 \pm 0.21$ & $1.51 \pm 0.14$ & $13.0 / 8$ & $0.67 \pm 0.11$ \\
$\mathrm{~W}$ & $230.5 \pm 10.2$ & $4.51 \pm 0.16$ & $1.68 \pm 0.12$ & $36.9 / 8$ & $0.69 \pm 0.08$ \\
\hline \multicolumn{5}{c}{$p A \rightarrow \phi X$} \\
\hline $\mathrm{C}$ & $7.6 \pm 0.8$ & $4.04 \pm 0.15$ & $1.26 \pm 0.13$ & $12.6 / 12$ & $0.66 \pm 0.09$ \\
$\mathrm{Ti}$ & $31.9 \pm 5.0$ & $3.81 \pm 0.21$ & $1.15 \pm 0.17$ & $10.5 / 12$ & $0.66 \pm 0.14$ \\
$\mathrm{~W}$ & $73.9 \pm 6.1$ & $4.20 \pm 0.14$ & $1.65 \pm 0.14$ & $28.4 / 12$ & $0.72 \pm 0.09$ \\
\hline \multicolumn{5}{c}{}
\end{tabular}

of the extrapolations is estimated by using parametrisation (7) as an alternative and taking the difference between the two approaches. Leaving out the bin of largest $p_{T}^{2}$ in the power-law fit increases the extrapolated $\phi$ cross section by about $6 \%$, which is fully compatible with this systematic uncertainty.

Taking into account the limited rapidity coverage of the detector, the integrated cross sections are then extrapolated to full phase space using target-specific correction factors $\Delta \sigma_{\text {vis }}$. These factors are derived from the MC simulators FRITIOF [40] and HIJING [49], and the average of both was taken for extrapolation. The systematic error was estimated by taking half the 
Table 4. Extrapolated total cross sections $\sigma_{p A}$, and integrated inclusive differential production cross sections $\sigma_{v i s}$ within the accessible phase space of the measurement. The errors denote the statistical error and the combined systematic error, respectively. The rapidity and $p_{T}^{2}$ coverage of the measurements are given in columns 3 and 4 . For the $\phi$ meson, $R_{p_{T}^{2}}^{e x t r}$ denotes the factor used for extrapolation of the integrated cross sections to $p_{T}^{2}=0$. $\Delta \sigma_{v i s}$ is the fraction of the total cross section within the rapidity coverage of the measurement, based on the FRITIOF [40] and HIJING [49] Monte-Carlo generators. Note that for these numbers the $p_{T}^{2}$-extrapolation for the $\phi$ cross sections was applied beforehand.

\begin{tabular}{|c|c|c|c|c|c|c|}
\hline & $\sigma_{p A}[\mathrm{mb}]$ & $\sigma_{v i s}[\mathrm{mb}]$ & rapidity interval & $p_{T}^{2}$ range $\left[\mathrm{GeV}^{2} / c^{2}\right]$ & $R_{p_{T}^{2}}^{\text {extr }}$ & $\Delta \sigma_{v i s}[\%]$ \\
\hline & & & \multicolumn{4}{|l|}{$p A \rightarrow K^{* 0} X$} \\
\hline $\mathrm{C}$ & $43.9 \pm 0.6 \pm 3.3$ & $12.1 \pm 0.2 \pm 0.9$ & \multirow[t]{3}{*}{$-0.8-+0.3$} & \multirow[t]{3}{*}{$0.0-12.0$} & 1 & $27.4 \pm 0.7$ \\
\hline $\mathrm{Ti}$ & $141.2 \pm 2.6 \pm 10.6$ & $38.5 \pm 0.7 \pm 2.7$ & & & 1 & $27.3 \pm 0.7$ \\
\hline W & $465.9 \pm 6.4 \pm 32.7$ & $127.5 \pm 1.7 \pm 8.3$ & & & 1 & $27.4 \pm 0.7$ \\
\hline & & & \multicolumn{4}{|l|}{$p A \rightarrow \bar{K}^{* 0} X$} \\
\hline $\mathrm{C}$ & $36.0 \pm 0.6 \pm 2.9$ & $10.0 \pm 0.2 \pm 0.7$ & \multirow[t]{3}{*}{$-0.8-+0.3$} & \multirow[t]{3}{*}{$0.0-12.0$} & 1 & $27.7 \pm 1.1$ \\
\hline $\mathrm{Ti}$ & $111.5 \pm 2.5 \pm 9.7$ & $31.1 \pm 0.7 \pm 2.3$ & & & 1 & $27.9 \pm 1.3$ \\
\hline \multirow[t]{2}{*}{ W } & $388.8 \pm 6.9 \pm 30.8$ & $107.6 \pm 1.9 \pm 7.0$ & & & 1 & $27.7 \pm 1.3$ \\
\hline & & & \multicolumn{4}{|l|}{$p A \rightarrow \phi X$} \\
\hline $\mathrm{C}$ & $11.0 \pm 0.2 \pm 1.4$ & $1.60 \pm 0.03 \pm 0.14$ & \multirow[t]{3}{*}{$-0.7-+0.25$} & \multirow[t]{3}{*}{$0.3-12.0$} & $1.94 \pm 0.13$ & $28.3 \pm 1.8$ \\
\hline $\mathrm{Ti}$ & $44.7 \pm 1.0 \pm 6.0$ & $6.4 \pm 0.1 \pm 0.6$ & & & $1.97 \pm 0.17$ & $28.3 \pm 1.7$ \\
\hline W & $135.1 \pm 1.6 \pm 17.0$ & $21.9 \pm 0.3 \pm 2.0$ & & & $1.72 \pm 0.14$ & $27.9 \pm 1.4$ \\
\hline
\end{tabular}

Table 5. Atomic mass number dependence of the integrated and total production cross sections, and extrapolated production cross sections in proton-nucleon reactions. The errors of $\alpha$ reflect the statistical errors and all targetmaterial dependent systematic errors. For $\sigma_{p N}$, full errors due to statistics and systematics are given. Errors were added in quadrature.

\begin{tabular}{lccc}
\hline & $p N \rightarrow K^{* 0} X$ & $p N \rightarrow \bar{K}^{* 0} X$ & $p N \rightarrow \phi X$ \\
\hline$\alpha_{\text {vis }}$ & $0.86 \pm 0.03$ & $0.87 \pm 0.03$ & $0.96 \pm 0.02$ \\
$\alpha_{p A}$ & $0.87 \pm 0.03$ & $0.87 \pm 0.03$ & $0.91 \pm 0.02$ \\
\hline$\sigma_{p N}[\mathrm{mb}]$ & $5.06 \pm 0.54$ & $4.02 \pm 0.45$ & $1.17 \pm 0.11$ \\
\hline
\end{tabular}

difference between the FRITIOF and the HIJING results. As quantified in Table 4 about $28 \%$ of the total cross section is accessible to the experiment. Correcting the limited phase space coverage leads to the total inclusive cross sections

$$
\sigma_{p A}=\frac{R_{p_{T}^{2}}^{e x t r} \cdot \sigma_{v i s}}{\Delta \sigma_{v i s}}
$$

for $K^{* 0}, \bar{K}^{* 0}$, and $\phi$ production in pC-, pTi-, and pW-interactions (collected in Table 4). The systematic error of the cross section extrapolation in total amounts to about $2.6 \%$ for the $K^{* 0}$, $4.5 \%$ for the $\bar{K}^{* 0}$, and $10.0 \%$ for the $\phi$ total production cross sections (Table 2).

\subsection{Atomic mass number dependence of the integrated and total cross sections}

Fig. 7 shows the atomic mass number dependence of the integrated cross sections $\sigma_{v i s}$ for the production of $K^{* 0}, \bar{K}^{* 0}$, and $\phi$ mesons in pA-interactions. The atomic number dependence is well-described by a power-law

$$
\sigma_{v i s}=\sigma_{v i s, 0} \cdot A^{\alpha_{v i s}}
$$

where $\sigma_{v i s, 0}$ is the visible proton-nucleon cross section. The results for the exponent $\alpha_{v i s}$ are collected in Table 5. Comparing the values with those for inelastic pA cross sections, $\alpha_{\text {inel }}=$ 


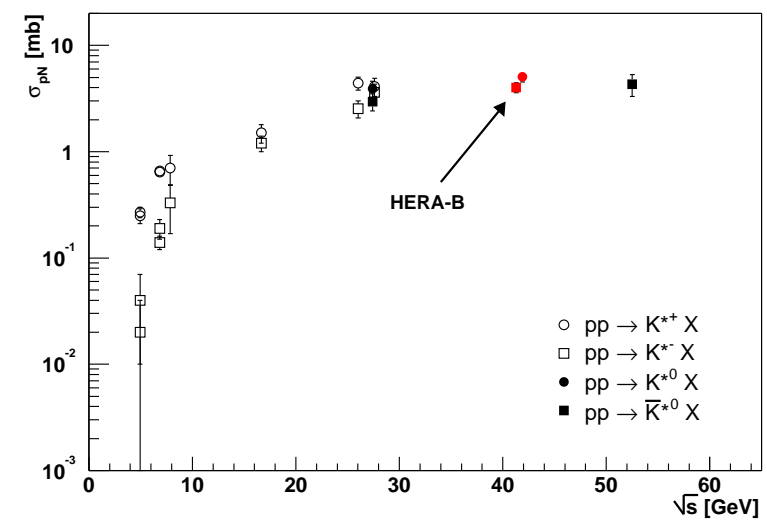

(a)

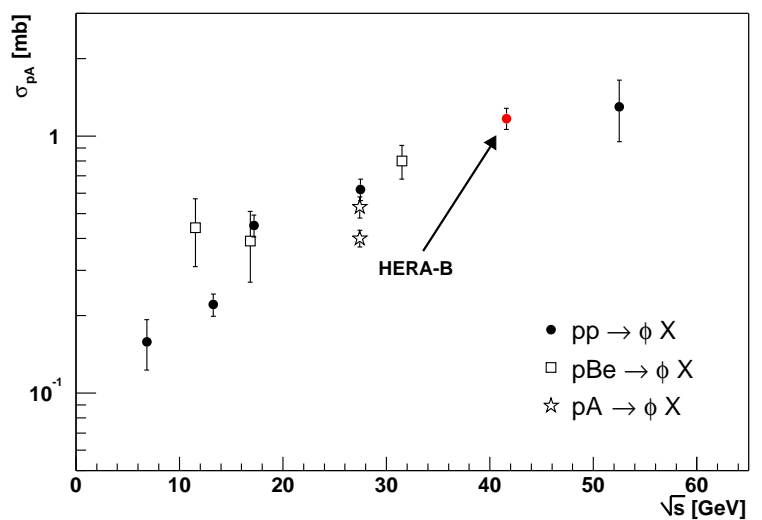

(b)

Figure 6. a) Comparison of $K^{* 0} / \bar{K}^{* 0}$ and $K^{* \pm}$ meson total production cross sections at various centre-of-mass energies [38,51-57]. The results of this analysis have been slightly displaced from each other for better visibility. b) same as a) but for $\phi$ meson production $[17,38,54,58-63]$. Results of the pBe measurements have been extrapolated to $A=1$ using $\alpha=0.91 \pm 0.02$ from [17].

$0.71 \pm 0.01[50]$, it follows that strange vector meson production shows a stronger $A$-dependence than the total inelastic cross section. While the latter scales roughly with the cross-sectional area of the nucleus, the $K^{* 0}$ and $\phi$ production cross sections show a tendency towards scaling with the volume.

The same parametrisation can be used to describe the $A$-dependence of the cross sections extrapolated to the full phase space,

$$
\sigma_{p A}=\sigma_{p N} \cdot A^{\alpha_{p A}}
$$

$\sigma_{p N}$ is an estimate of the total cross section for strange vector meson production in protonnucleon reactions. The fitted values for $\alpha_{p A}$ and $\sigma_{p N}$ are included in Table 5, and the results for $\sigma_{p N}$ are compared to those measured at other energies in pp and pA reactions (Fig. 66). Recently, the NA60 collaboration measured the production of $\phi$ mesons in collisions of $400 \mathrm{GeV}$ protons with various nuclei [17]. Fitting their data to eq. 12, they obtain $\alpha=0.91 \pm 0.02$, which is in good agreement with our measurements. Consequently, the pBe data displayed in Fig. 6 b) were rescaled using this result.

\subsection{Cronin effect}

The $A$-dependence of the differential cross sections $d \sigma / d y$ and $d \sigma / d p_{T}^{2}$ themselves is also of interest. Fig. 8 demonstrates that, for the differential cross sections integrated over $p_{T}^{2}$, the exponent $\alpha$ is in good approximation independent of rapidity in the range of the measurement. Note that mainly soft interactions with $p_{T}^{2} \leq 1 \mathrm{GeV}^{2} / \mathrm{c}^{2}$ contribute to the cross section.

In contrast, $\alpha$ depends on the transverse momentum. With increasing $p_{T}^{2}, \alpha$ constantly increases, as shown in Fig. 9. Cronin et al. [21] have observed this effect for the production of stable particles $(\pi, K, p)$. In this analysis we report an observation of the Cronin effect for the production of vector mesons containing strange quarks. Note however, that the exponent for $K^{* 0}$ and $\bar{K}^{* 0}$ production is smaller than that for $K^{+}$and $K^{-}$mesons. Only at the highest $p_{T}$ values do the distributions get close to each other, while the exponents $\alpha$ for $\phi$ and $K^{+}$mesons coincide for all transverse momenta.

Qualitatively the observations shown in Fig. 9 are in agreement with the model of [25] which predicts a decrease of $\alpha$ with increasing CMS energy; this is indeed observed for the $K^{* 0}\left(\bar{K}^{* 0}\right)$ 


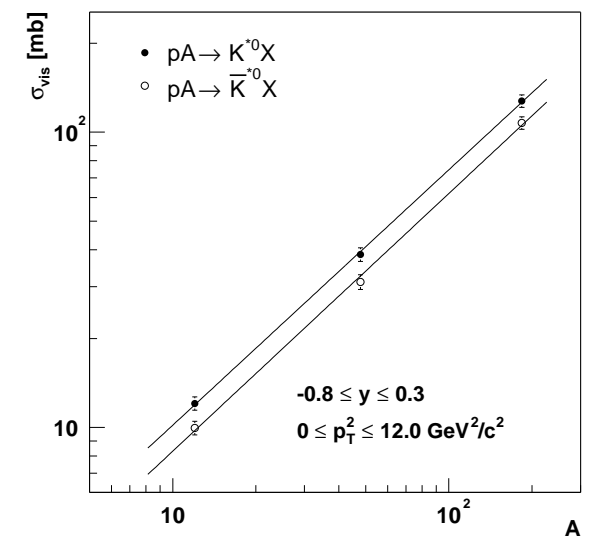

(a)

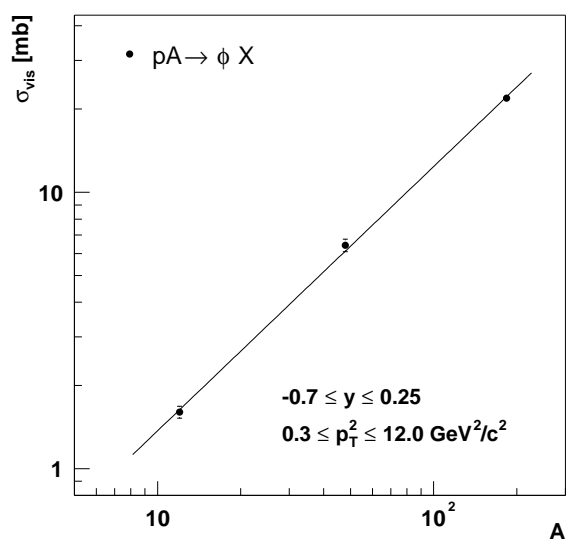

(b)

Figure 7. Atomic mass number dependence of the integrated inclusive cross sections for a) $K^{* 0} / \bar{K}^{* 0}$ and b) $\phi$ production. Fits to the parametrisation (11) are superimposed. The errors shown reflect the statistical error as well as all target-material dependent systematic errors, added in quadrature.

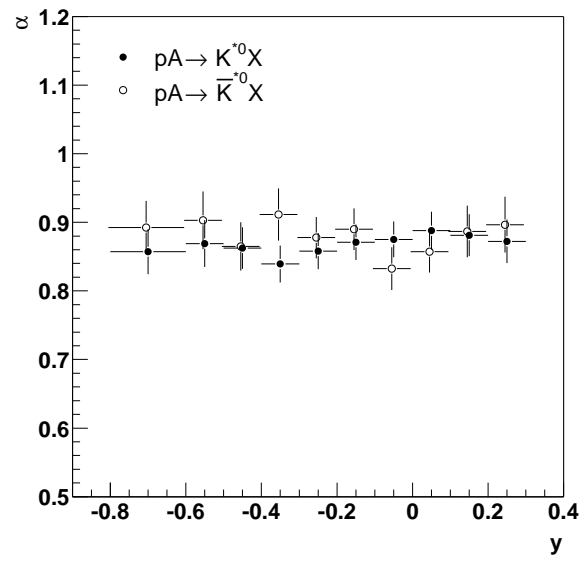

(a)

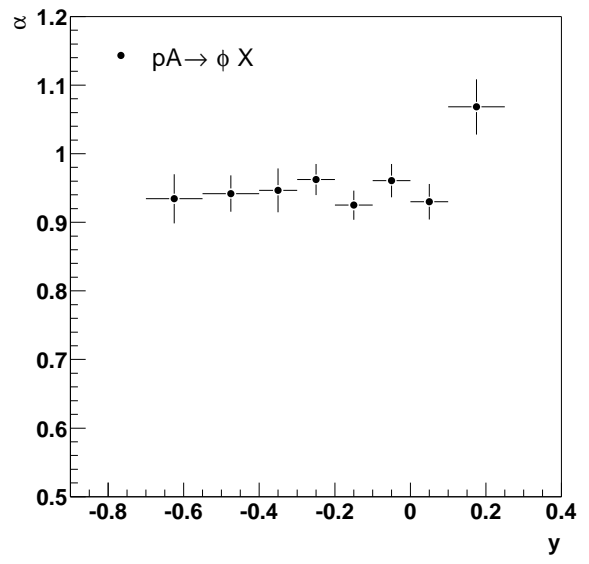

(b)

Figure 8. Measured values of $\alpha(y)$ for a) $K^{* 0} / \bar{K}^{* 0}$ and b) $\phi$ production.

data collected at a beam energy of $920 \mathrm{GeV}$ when compared to the $K^{+}\left(K^{-}\right)$results of Cronin et al. measured at $400 \mathrm{GeV}$. On the other hand $\alpha_{\phi}$ produced at a beam energy of $920 \mathrm{GeV}$ coincides with that of $K^{+}$mesons collected at $400 \mathrm{GeV}$; this might indicate a different flavor composition of the initially scattered partons fragmenting to $\phi$ mesons as compared to $K^{* 0}\left(\bar{K}^{* 0}\right)$ mesons.

\section{Conclusion}

The cross sections of $K^{* 0}, \bar{K}^{* 0}$, and $\phi$ meson production in the central rapidity region have been measured for the first time in pC-, pTi-, and pW-interactions. The cross sections show a power-law dependence $\sigma \propto A^{\alpha}$ with an exponent of $\alpha \approx 0.8-1.1$ depending both on the produced vector meson and the transverse momentum. The exponent $\alpha$ is found to be larger than the corresponding value for the total inelastic cross section. The measurements of $\alpha\left(p_{T}^{2}\right)$ 

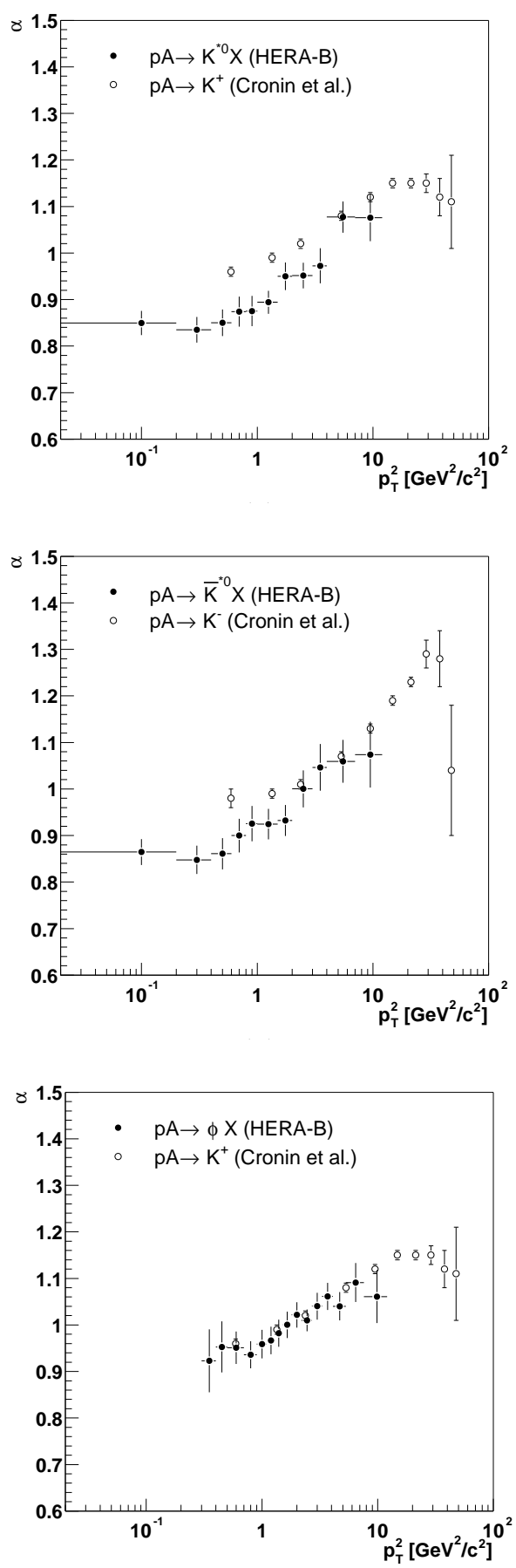

(c)

Figure 9. Measured values of $\alpha\left(p_{T}\right)$ for a) $K^{* 0}$, b) $\bar{K}^{* 0}$ and c) $\phi$ production. The results of Cronin et al. [21] on the $K^{ \pm}$mesons are superimposed. Numbers of this analysis are summarised in Table 7

for the $\phi$ vector meson agree with the values determined by Cronin et al. [21] for $K^{+}$mesons. For the $K^{* 0} / \bar{K}^{* 0}$ mesons, $\alpha\left(p_{T}^{2}\right)$ is systematically smaller. 


\section{Acknowledgements}

We thank B. Kopeliovich for helpful comments and discussions about the interpretation of our results. We express our gratitude to the DESY laboratory for the strong support in setting up and running the HERA-B experiment. We are also indebted to the DESY accelerator group for the continuous efforts to provide good and stable beam conditions. The HERA-B experiment would not have been possible without the enormous effort and commitment of our technical and administrative staff. It is a pleasure to thank all of them.

\section{References}

1. C.Y. Wong, Introduction to High Energy Heavy-Ion Collisions (World Scientific, Singapore), 516 (1994)

2. H. Satz, Nucl. Phys. Proc. Suppl. 94, 204 (2001)

3. J. Letessier and J. Rafelski, Int. J. Mod. Phys. E 9, 107 (2000)

4. C. Markert et al., hep-ph/0206260 (2002)

5. J. Rafelski et al., Phys. Rev. C 64, 054907 (2001)

6. C. Markert, J. Phys. G: Nucl. Part. Phys 31, S169 (2005)

7. P. Jacobs and X.N. Wang, Progr. Part. Nucl. Phys. 54, 443 (2005)

8. H. Zhang, nucl-ex/0403010 (2004)

9. J. Adams et al., Phys. Rev. C 71, 064902 (2005)

10. C. Adler et al., Phys. Rev. C 65, 041901 (2002)

11. J. Adams et. al., Phys. Lett. B 612, 181 (2005)

12. S.S. Adler et al., Phys. Rev. C 72, 014903 (2005)

13. S.S. Adler et al., Eur. Phys. J. C 43, 317 (2005)

14. D. Pal, Nucl. Phys. A 774, 489 (2006)

15. S.L. Blyth, nucl-ex/0608019 (2006)

16. Y.G. Ma, nucl-ex/0609020 (2006)

17. H.K. Wöhri et al., Eur. Phys. J C 43, 407 (2005)

18. R. Bellwied, J. Phys. G: Nucl.Part. Phys. 31, S675 (2005)

19. X. Cai, J. Phys. G: Nucl. Part. Phys. 31, S1015 (2005)

20. J. Cronin et al., Phys. Rev. D 11, 3105 (1975)

21. J. Cronin et al., Phys. Rev. Lett. 38, 670 (1977)

22. For a recent review, see A. Accardi, Acta Phys Hung. A 22, 289 (2005)

23. A. Accardi, Eur. Phys. J. C 43, 121 (2005)

24. R.C. Hwa and C.B. Yang, Phys. Rev. Lett. 93, 082302 (2004)

25. B.Z. Kopeliovich et al., Phys. Rev. Lett. 88, 232303 (2002)

26. K. Ehret et al., Nucl Instr. Meth. A 446, 190 (2000)

27. C. Bauer et al., Nucl. Instr. Meth. A 501, 39 (2003)

28. H. Albrecht et al., Nucl. Instr. Meth. A 555, 319 (2005); H. Albrecht et al., Nucl. Instr. Meth. A 541, 610 (2005)

29. I. Ariño et al., Nucl. Instr. Meth. A 516, 445 (2004)

30. G. Avoni et al., Nucl. Instr. Meth A 461, 332 (2001)

31. I. Abt et al., Phys. Lett. B 596, 173 (2004)

32. I. Abt et al., Luminosity determination at HERA-B, to be published

33. I. Abt et al., Nucl. Instr. Meth. A 490, 546 (2002)

34. M. Symalla, PhD thesis, University of Dortmund; DESY-THESIS-2004-020 (2004)

35. C. van Eldik, PhD thesis, University of Dortmund; DESY-THESIS-2004-019 (2004)

36. J.D. Jackson, Nuov. Cim. 34, 1644 (1964)

37. S. Eidelman et al., Phys. Lett. B 592, 1 (2004)

38. M. Aguilar-Benitez et al., Z. Phys. C 50, 405 (1991)

39. P. Granet et al., Nucl. Phys. B 140, 389 (1978)

40. H. Pi, Comp. Phys. Comm. 71, 173 (1992)

41. R. Brun et al., GEANT3, CERN-DD-EE-84-1 (1987)

42. H. Albrecht et al., Z. Phys. C 61, 1 (1994)

43. R. Pernack, PhD thesis, University of Rostock (2004)

44. R.P. Feynman, Phys. Rev. Lett. 23, 1415 (1969)

45. W.M. Geist et al., Phys. Rep. 197, 263 (1990)

46. A. Breakstone et al., Phys. Lett. B 135, 510 (1984)

47. A. Breakstone et al., Z. Phys. C 27, 205 (1985) 
Table 6. Compilation of the differential production cross sections $d \sigma / d y$ in the accessible phase space of the measurement. The errors reflect the statistical and full systematic errors, respectively.

\begin{tabular}{|c|c|c|c|c|}
\hline & \multirow[t]{2}{*}{$\Delta y$} & \multicolumn{3}{|c|}{$d \sigma / d y[\mathrm{mb}]$} \\
\hline & & $\mathrm{C}$ & $\mathrm{Ti}$ & W \\
\hline$K^{* 0}$ & $\begin{array}{c}-0.8--0.6 \\
-0.6--0.5 \\
-0.5--0.4 \\
-0.4--0.3 \\
-0.3--0.2 \\
-0.2--0.1 \\
-0.1-0.0 \\
0.0-+0.1 \\
+0.1-+0.2 \\
+0.2-+0.3 \\
\end{array}$ & $\begin{array}{l}12.5 \pm 0.4 \pm 0.9 \\
12.3 \pm 0.5 \pm 0.9 \\
10.8 \pm 0.4 \pm 0.8 \\
12.2 \pm 0.3 \pm 0.9 \\
11.1 \pm 0.3 \pm 0.8 \\
10.6 \pm 0.3 \pm 0.7 \\
10.7 \pm 0.3 \pm 0.7 \\
11.1 \pm 0.3 \pm 0.8 \\
10.1 \pm 0.3 \pm 0.7 \\
10.0 \pm 0.4 \pm 0.8\end{array}$ & $\begin{array}{l}38.6 \pm 2.2 \pm 2.9 \\
41.6 \pm 2.2 \pm 3.2 \\
34.0 \pm 1.8 \pm 2.5 \\
39.7 \pm 1.7 \pm 2.9 \\
34.4 \pm 1.7 \pm 2.4 \\
33.5 \pm 1.4 \pm 2.3 \\
34.6 \pm 1.4 \pm 2.4 \\
33.5 \pm 1.5 \pm 2.4 \\
32.4 \pm 1.5 \pm 2.4 \\
37.0 \pm 2.3 \pm 2.8\end{array}$ & $\begin{array}{l}128.3 \pm 4.6 \pm 8.6 \\
131.6 \pm 5.4 \pm 9.0 \\
112.9 \pm 3.7 \pm 7.3 \\
120.6 \pm 3.4 \pm 7.7 \\
114.3 \pm 3.0 \pm 7.1 \\
112.8 \pm 3.0 \pm 7.0 \\
115.2 \pm 2.9 \pm 7.1 \\
121.2 \pm 3.3 \pm 7.6 \\
110.3 \pm 3.6 \pm 7.2 \\
109.0 \pm 3.9 \pm 7.5\end{array}$ \\
\hline & $\begin{array}{c}-0.8--0.6 \\
-0.6--0.5 \\
-0.5--0.4 \\
-0.4--0.3 \\
-0.3--0.2 \\
-0.2--0.1 \\
-0.1-0.0 \\
0.0-+0.1 \\
+0.1-+0.2 \\
+0.2-+0.3 \\
\end{array}$ & $\begin{array}{l}8.5 \pm 0.4 \pm 0.7 \\
9.9 \pm 0.5 \pm 0.8 \\
9.2 \pm 0.4 \pm 0.7 \\
8.8 \pm 0.4 \pm 0.7 \\
9.4 \pm 0.3 \pm 0.7 \\
9.3 \pm 0.3 \pm 0.7 \\
9.5 \pm 0.3 \pm 0.7 \\
8.7 \pm 0.3 \pm 0.6 \\
8.9 \pm 0.3 \pm 0.7 \\
8.1 \pm 0.4 \pm 0.7 \\
\end{array}$ & $\begin{array}{l}28.7 \pm 1.7 \pm 2.3 \\
30.0 \pm 2.1 \pm 2.5 \\
31.5 \pm 1.8 \pm 2.5 \\
26.6 \pm 1.6 \pm 2.1 \\
31.1 \pm 1.5 \pm 2.3 \\
31.5 \pm 1.8 \pm 2.4 \\
28.4 \pm 1.5 \pm 2.1 \\
26.0 \pm 1.3 \pm 1.9 \\
25.1 \pm 1.6 \pm 2.0 \\
25.9 \pm 1.8 \pm 2.2 \\
\end{array}$ & $\begin{array}{c}96.8 \pm 4.1 \pm 7.1 \\
114.0 \pm 4.9 \pm 8.6 \\
97.7 \pm 4.3 \pm 6.9 \\
102.9 \pm 4.0 \pm 7.4 \\
102.8 \pm 3.4 \pm 6.7 \\
105.6 \pm 3.5 \pm 7.0 \\
91.7 \pm 3.3 \pm 6.1 \\
88.8 \pm 3.4 \pm 5.6 \\
97.3 \pm 3.2 \pm 7.2 \\
92.3 \pm 4.0 \pm 7.1 \\
\end{array}$ \\
\hline$\phi$ & $\begin{array}{c}-0.70--0.55 \\
-0.55--0.40 \\
-0.40--0.30 \\
-0.30--0.20 \\
-0.20--0.10 \\
-0.10-0.00 \\
0.00-+0.10 \\
+0.10-+0.25 \\
\end{array}$ & $\begin{array}{l}1.77 \pm 0.10 \pm 0.18 \\
1.81 \pm 0.06 \pm 0.17 \\
1.80 \pm 0.06 \pm 0.21 \\
1.60 \pm 0.05 \pm 0.14 \\
1.71 \pm 0.05 \pm 0.14 \\
1.64 \pm 0.05 \pm 0.14 \\
1.84 \pm 0.07 \pm 0.16 \\
1.45 \pm 0.10 \pm 0.14\end{array}$ & $\begin{array}{l}7.2 \pm 0.6 \pm 0.8 \\
7.0 \pm 0.4 \pm 0.7 \\
7.2 \pm 0.3 \pm 0.7 \\
6.8 \pm 0.3 \pm 0.7 \\
6.7 \pm 0.3 \pm 0.6 \\
6.4 \pm 0.3 \pm 0.6 \\
7.3 \pm 0.4 \pm 0.7 \\
6.1 \pm 0.5 \pm 0.6 \\
\end{array}$ & $\begin{array}{l}23.1 \pm 0.9 \pm 2.1 \\
24.0 \pm 0.6 \pm 2.1 \\
24.7 \pm 0.7 \pm 2.2 \\
23.0 \pm 0.5 \pm 2.0 \\
21.7 \pm 0.5 \pm 1.9 \\
22.7 \pm 0.6 \pm 2.0 \\
23.6 \pm 0.8 \pm 2.1 \\
26.5 \pm 1.1 \pm 2.5\end{array}$ \\
\hline
\end{tabular}

48. V.V. Abramov et al., Sov. J. Nucl. Phys. 41, 445 (1985)

49. X.N. Wang and M. Gyulassy, Phys. Rev. D 44, 3501 (1991)

50. W.M. Geist et al., Nucl. Phys. A 525, 143c (1991)

51. V. Blobel et al., Phys. Lett. B 48, 73 (1974)

52. K. Bockmann et al., Nucl. Phys. B 166, 284 (1979)

53. H. Kichimi et al., Lett. Nuov. Cim. 24, 129 (1979)

54. D. Drijard et al., Z. Phys. C 9, 293 (1981)

55. D. Brick et al., Phys. Rev. D 25, 2248 (1982)

56. T. Aziz et al., Z. Phys. C 30, 381 (1986)

57. M.Y. Bogolyubsky et al., IFVE-89-5 (1988)

58. T. Sammer, PhD thesis, Munich (2001)

59. S. Kartik, PhD thesis, Indiana University (1991)

60. C. Daum et al., Nucl. Phys. B 186, 205 (1981)

61. V. Blobel et al., Phys. Lett. B 59, 88 (1975)

62. Yu. M. Antipov et al., Phys. Lett. B 110, 326 (1982)

63. K.J. Anderson et al., Phys. Rev. Lett. 37, 799 (1976) 
Table 7. Compilation of the differential production cross sections $d \sigma / d p_{T}^{2}$ in the accessible phase space of the measurement. The errors reflect the statistical and full systematic errors, respectively. The right column lists the results of the $A^{\alpha}$ fits shown in Figs. 9. The errors given are the combined statistical and target-material dependent systematic errors, added in quadrature.

\begin{tabular}{|c|c|c|c|c|c|}
\hline & \multirow[t]{2}{*}{$\Delta\left(p_{T}^{2}\right)\left[\mathrm{GeV}^{2} / \mathrm{c}^{2}\right]$} & \multicolumn{4}{|c|}{$d \sigma / d p_{T}^{2}\left[\mathrm{mb} /(\mathrm{GeV} / \mathrm{c})^{2}\right]$} \\
\hline & & $\mathrm{C}$ & $\mathrm{Ti}$ & $\mathrm{W}$ & $\alpha$ \\
\hline$K^{* 0}$ & $\begin{array}{l}0.0-0.2 \\
0.2-0.4 \\
0.4-0.6 \\
0.6-0.8 \\
0.8-1.0 \\
1.0-1.5 \\
1.5-2.0 \\
2.0-3.0 \\
3.0-4.0 \\
4.0-7.0 \\
7.0-12.0 \\
\end{array}$ & $\begin{array}{c}22.2 \pm 0.5 \pm 1.5 \\
12.2 \pm 0.4 \pm 0.8 \\
7.4 \pm 0.2 \pm 0.5 \\
4.7 \pm 0.2 \pm 0.3 \\
3.5 \pm 0.1 \pm 0.3 \\
1.92 \pm 0.04 \pm 0.13 \\
0.91 \pm 0.03 \pm 0.07 \\
0.37 \pm 0.01 \pm 0.03 \\
0.157 \pm 0.006 \pm 0.013 \\
0.035 \pm 0.001 \pm 0.003 \\
0.0044 \pm 0.0003 \pm 0.0004 \\
\end{array}$ & $\begin{array}{c}69.4 \pm 4.3 \pm 4.9 \\
38.9 \pm 1.6 \pm 2.7 \\
25.5 \pm 1.3 \pm 1.8 \\
14.5 \pm 0.8 \pm 1.1 \\
10.6 \pm 0.7 \pm 0.8 \\
6.4 \pm 0.2 \pm 0.4 \\
3.1 \pm 0.2 \pm 0.2 \\
1.45 \pm 0.06 \pm 0.11 \\
0.56 \pm 0.04 \pm 0.05 \\
0.142 \pm 0.008 \pm 0.011 \\
0.021 \pm 0.002 \pm 0.002 \\
\end{array}$ & $\begin{array}{c}224.3 \pm 5.8 \pm 14.0 \\
119.4 \pm 4.2 \pm 7.4 \\
76.0 \pm 2.7 \pm 4.9 \\
50.5 \pm 2.0 \pm 3.3 \\
37.9 \pm 1.3 \pm 2.5 \\
21.8 \pm 0.5 \pm 1.3 \\
11.9 \pm 0.3 \pm 0.8 \\
5.0 \pm 0.1 \pm 0.3 \\
2.20 \pm 0.08 \pm 0.16 \\
0.64 \pm 0.02 \pm 0.04 \\
0.084 \pm 0.004 \pm 0.007 \\
\end{array}$ & $\begin{array}{l}0.85 \pm 0.03 \\
0.84 \pm 0.03 \\
0.85 \pm 0.03 \\
0.87 \pm 0.03 \\
0.88 \pm 0.03 \\
0.89 \pm 0.02 \\
0.95 \pm 0.03 \\
0.95 \pm 0.03 \\
0.97 \pm 0.04 \\
1.08 \pm 0.03 \\
1.08 \pm 0.05 \\
\end{array}$ \\
\hline $\bar{K}^{* 0}$ & $\begin{array}{l}0.0-0.2 \\
0.2-0.4 \\
0.4-0.6 \\
0.6-0.8 \\
0.8-1.0 \\
1.0-1.5 \\
1.5-2.0 \\
2.0-3.0 \\
3.0-4.0 \\
4.0-7.0 \\
7.0-12.0 \\
\end{array}$ & $\begin{array}{c}19.3 \pm 0.5 \pm 1.4 \\
10.1 \pm 0.3 \pm 0.7 \\
6.2 \pm 0.2 \pm 0.4 \\
4.0 \pm 0.2 \pm 0.3 \\
2.8 \pm 0.1 \pm 0.2 \\
1.55 \pm 0.04 \pm 0.12 \\
0.68 \pm 0.02 \pm 0.05 \\
0.264 \pm 0.010 \pm 0.022 \\
0.102 \pm 0.006 \pm 0.011 \\
0.024 \pm 0.001 \pm 0.002 \\
0.0018 \pm 0.0002 \pm 0.0002 \\
\end{array}$ & $\begin{array}{c}59.2 \pm 3.4 \pm 4.3 \\
31.0 \pm 1.5 \pm 2.2 \\
19.1 \pm 1.1 \pm 1.4 \\
13.9 \pm 0.9 \pm 1.1 \\
10.1 \pm 0.7 \pm 0.8 \\
5.1 \pm 0.3 \pm 0.4 \\
2.6 \pm 0.1 \pm 0.2 \\
0.88 \pm 0.05 \pm 0.07 \\
0.45 \pm 0.03 \pm 0.04 \\
0.097 \pm 0.008 \pm 0.009 \\
0.010 \pm 0.002 \pm 0.001 \\
\end{array}$ & $\begin{array}{c}201.8 \pm 5.5 \pm 13.0 \\
101.3 \pm 3.7 \pm 6.5 \\
65.0 \pm 2.9 \pm 4.3 \\
46.2 \pm 2.3 \pm 3.2 \\
34.5 \pm 1.7 \pm 2.5 \\
19.0 \pm 0.7 \pm 1.3 \\
8.7 \pm 0.3 \pm 0.6 \\
3.9 \pm 0.1 \pm 0.3 \\
1.78 \pm 0.08 \pm 0.16 \\
0.42 \pm 0.02 \pm 0.03 \\
0.035 \pm 0.003 \pm 0.003 \\
\end{array}$ & $\begin{array}{l}0.86 \pm 0.03 \\
0.85 \pm 0.03 \\
0.86 \pm 0.03 \\
0.90 \pm 0.04 \\
0.93 \pm 0.04 \\
0.92 \pm 0.03 \\
0.93 \pm 0.03 \\
1.00 \pm 0.04 \\
1.05 \pm 0.05 \\
1.06 \pm 0.05 \\
1.07 \pm 0.07 \\
\end{array}$ \\
\hline$\phi$ & $\begin{array}{l}0.3-0.4 \\
0.4-0.5 \\
0.5-0.7 \\
0.7-0.9 \\
0.9-1.1 \\
1.1-1.3 \\
1.3-1.5 \\
1.5-1.8 \\
1.8-2.2 \\
2.2-2.7 \\
2.7-3.3 \\
3.3-4.1 \\
4.1-5.3 \\
5.3-7.6 \\
7.6-12.0\end{array}$ & $\begin{array}{c}2.8 \pm 0.3 \pm 0.2 \\
2.3 \pm 0.2 \pm 0.3 \\
1.70 \pm 0.09 \pm 0.16 \\
1.09 \pm 0.05 \pm 0.09 \\
0.73 \pm 0.03 \pm 0.07 \\
0.46 \pm 0.02 \pm 0.04 \\
0.35 \pm 0.02 \pm 0.03 \\
0.266 \pm 0.010 \pm 0.024 \\
0.163 \pm 0.006 \pm 0.015 \\
0.098 \pm 0.001 \pm 0.009 \\
0.058 \pm 0.002 \pm 0.005 \\
0.031 \pm 0.001 \pm 0.003 \\
0.0157 \pm 0.0008 \pm 0.0014 \\
0.0049 \pm 0.0003 \pm 0.0005 \\
0.0010 \pm 0.0001 \pm 0.0001\end{array}$ & $\begin{array}{c}9.4 \pm 1.7 \pm 1.2 \\
9.2 \pm 1.3 \pm 1.1 \\
7.0 \pm 0.6 \pm 0.7 \\
4.5 \pm 0.3 \pm 0.5 \\
2.8 \pm 0.2 \pm 0.3 \\
2.0 \pm 0.1 \pm 0.2 \\
1.57 \pm 0.09 \pm 0.16 \\
1.02 \pm 0.06 \pm 0.10 \\
0.62 \pm 0.03 \pm 0.06 \\
0.43 \pm 0.02 \pm 0.04 \\
0.24 \pm 0.01 \pm 0.02 \\
0.145 \pm 0.009 \pm 0.014 \\
0.065 \pm 0.005 \pm 0.007 \\
0.021 \pm 0.002 \pm 0.002 \\
0.0063 \pm 0.0010 \pm 0.0009\end{array}$ & $\begin{array}{c}34.9 \pm 4.1 \pm 3.8 \\
31.2 \pm 2.5 \pm 3.1 \\
23.0 \pm 1.1 \pm 2.1 \\
14.2 \pm 0.6 \pm 1.3 \\
10.1 \pm 0.4 \pm 0.9 \\
6.6 \pm 0.2 \pm 0.6 \\
5.2 \pm 0.2 \pm 0.5 \\
4.0 \pm 0.1 \pm 0.4 \\
2.59 \pm 0.07 \pm 0.23 \\
1.57 \pm 0.04 \pm 0.14 \\
0.99 \pm 0.03 \pm 0.09 \\
0.57 \pm 0.02 \pm 0.05 \\
0.268 \pm 0.010 \pm 0.024 \\
0.095 \pm 0.004 \pm 0.009 \\
0.0183 \pm 0.0008 \pm 0.0020\end{array}$ & $\begin{array}{l}0.92 \pm 0.07 \\
0.95 \pm 0.06 \\
0.95 \pm 0.03 \\
0.94 \pm 0.03 \\
0.96 \pm 0.03 \\
0.97 \pm 0.03 \\
0.98 \pm 0.03 \\
1.00 \pm 0.03 \\
1.02 \pm 0.03 \\
1.01 \pm 0.02 \\
1.04 \pm 0.03 \\
1.06 \pm 0.03 \\
1.04 \pm 0.03 \\
1.09 \pm 0.04 \\
1.06 \pm 0.06\end{array}$ \\
\hline
\end{tabular}

